\title{
Article
}

\section{Admissibility Conjecture and Kazhdan's Property (T) for quantum groups}

Das, Biswarup, Daws, Matthew and Salmi, Pekka

Available at http://clok.uclan.ac.uk/25869/

Das, Biswarup, Daws, Matthew ORCID: 0000-0003-1707-4308 and Salmi, Pekka (2019) Admissibility Conjecture and Kazhdan's Property (T) for quantum groups. Journal of Functional Analysis, 276 (11). pp. 3484-3510. ISSN 00221236

It is advisable to refer to the publisher's version if you intend to cite from the work. http://dx.doi.org/10.1016/j.jfa.2018.09.001

For more information about UCLan's research in this area go to

http://www.uclan.ac.uk/researchgroups/ and search for < name of research Group>.

For information about Research generally at UCLan please go to http://www.uclan.ac.uk/research/

All outputs in CLoK are protected by Intellectual Property Rights law, including Copyright law. Copyright, IPR and Moral Rights for the works on this site are retained by the individual authors and/or other copyright owners. Terms and conditions for use of this material are defined in the policies page. 


\title{
ADMISSIBILITY CONJECTURE AND KAZHDAN'S PROPERTY (T) FOR QUANTUM GROUPS
}

\author{
BISWARUP DAS, MATTHEW DAWS, AND PEKKA SALMI
}

\begin{abstract}
We give a partial solution to a long-standing open problem in the theory of quantum groups, namely we prove that all finite-dimensional representations of a wide class of locally compact quantum groups factor through matrix quantum groups (Admissibility Conjecture for quantum group representations). We use this to study Kazhdan's Property (T) for quantum groups with non-trivial scaling group, strengthening and generalising some of the earlier results obtained by Fima, Kyed and Soltan, Chen and Ng, Daws, Skalski and Viselter, and Brannan and Kerr. Our main results are:

(i) All finite-dimensional unitary representations of locally compact quantum groups which are either unimodular or arise through a special bicrossed product construction are admissible.

(ii) A generalisation of a theorem of Wang which characterises Property ( $\mathrm{T}$ ) in terms of isolation of finite-dimensional irreducible representations in the spectrum.

(iii) A very short proof of the fact that quantum groups with Property (T) are unimodular.

(iv) A generalisation of a quantum version of a theorem of Bekka-Valette proven earlier for quantum groups with trivial scaling group, which characterises Property $(\mathrm{T})$ in terms of non-existence of almost invariant vectors for weakly mixing representations.

(v) A generalisation of a quantum version of Kerr-Pichot theorem, proven earlier for quantum groups with trivial scaling group, which characterises Property (T) in terms of denseness properties of weakly mixing representations.
\end{abstract}

\section{INTRODUCTION}

Property (T) was introduced in the mid-1960s by Kazhdan, as a tool to demonstrate that a large class of lattices are finitely generated. The discovery of Property $(\mathrm{T})$ was a cornerstone in group theory and the last decade saw its importance in many different subjects like ergodic theory, abstract harmonic analysis, operator algebras and some of the very recent topics like $\mathrm{C}^{*}$-tensor categories (see [8, 11, 37, 34] and references therein). In the late 1980s the subject of operator algebraic quantum groups gained prominence starting with the seminal work of Woronowicz [4], followed by works of Baaj, Skandalis, Woronowicz, Van Daele, Kustermans, Vaes and others [3, 46, 47, 27, 31]. Quantum groups can be looked upon as noncommutative analogues of locally compact groups, so quite naturally the notion of Property $(\mathrm{T})$ appeared also in that more general context. Property $(\mathrm{T})$ was first studied within the framework of Kac algebras (a precursor to the theory of locally compact quantum groups) [35], then for algebraic quantum groups [5] and discrete quantum groups [22, 29], and more recently for locally compact quantum groups [10, 18, 9].

By definition a locally compact group $G$ has Property (T) if every unitary representation with approximately invariant vectors has in fact a non-zero invariant vector. This definition extends verbatim to locally compact quantum groups, using the natural extensions of the necessary terms. By a result of Fima, a discrete quantum group having Property (T) is necessarily a Kac algebra, which is equivalent to being unimodular in the case of discrete 
quantum groups, and is the dual of a compact matrix quantum group [22, Propositions 7 \& 8]. This is a quantum generalisation of a result originally due to Kazhdan 8, Theorem 1.3 .1 \& Corollary 1.3.6]. In particular, while studying Property $(\mathrm{T})$ for discrete quantum groups, one is lead to consider only unimodular discrete quantum groups. Since a discrete quantum group is unimodular if and only if it is of Kac type, unimodular discrete quantum groups have trivial scaling automorphism groups, and this is important in what follows.

Generalising to locally compact quantum groups, Brannan and Kerr proved that a second countable locally compact quantum group with Property $(\mathrm{T})$ is necessarily unimodular [9, Theorem 6.3] - a result for which we will also give a new and short proof without the second countability assumption. So again while studying Property (T) for quantum groups, one is lead to consider only unimodular quantum groups. However, a unimodular locally compact quantum group can have a non-trivial scaling automorphism group. Examples of such locally compact quantum groups are Drinfeld doubles of non-Kac-type compact quantum groups: see Section 4.2. A recentresult of Arano (see [1, Theorem 7.5]), which finds applications in the study of $\mathrm{C}^{*}$-tensor categories and subfactors [34, 37], states that the Drinfeld double of the Woronowicz compact quantum group $S U_{q}(2 n+1)$ has Property $(\mathrm{T})$. This produces a concrete example of a unimodular locally compact quantum group with non-trivial scaling automorphism group, which has Property (T).

In this paper, we study Property $(\mathrm{T})$ and related problems, in particular on unimodular locally compact quantum groups with non-trivial scaling automorphism group. To enable this study, we prove the 'Admissibility Conjecture' for unimodular locally compact quantum groups, that is, we show that every finite-dimensional unitary representation of a unimodular locally compact quantum group is admissible. The Admissibility Conjecture is a long-standing open problem in the theory of quantum groups, which was implicitly stated in [39] and was conjectured in [15, Conjecture 7.2]. Admissibility of a finite-dimensional unitary representation of a quantum group means effectively that it 'factors' through a compact matrix quantum group.

Returning to locally compact groups, we note the following important characterisation of Property (T) by Bekka and Valette [7, Theorem 1]: a locally compact group $G$ has Property (T) if and only if every unitary representation of $G$ with approximately invariant vectors is not weakly mixing (i.e. admits a non-zero finite-dimensional subrepresentation). This characterisation turns out to be more useful from the application perspective than the definition itself, as has been elucidated in [9]. An important consequence of the Bekka-Valette theorem is the Kerr-Pichot theorem which states that if $G$ does not have Property (T), then within the set of all unitary representations on a fixed separable Hilbert space, the weakly mixing ones form a dense $G_{\delta}$-set in the weak topology, strengthening an earlier result of Glasner and Weiss [23, Theorem $2^{\prime}$ ] concerning the density of ergodic representations. Another important result along characterising Property $(\mathrm{T})$ is that $G$ has Property $(\mathrm{T})$ if and only if the trivial representation is isolated in the hull-kernel topology of the dual space $\widehat{G}$ [44]. A theorem of Wang [44, Theorem 2.1] (see also [8, Theorem 1.2.5]) extends this to all irreducible finitedimensional unitary representations of $G$ i.e. $G$ has Property (T) if and only if all irreducible finite-dimensional unitary representations of $G$ are isolated in $\widehat{G}$. This in particular helps us better understand the structure of the full group $\mathrm{C}^{*}$-algebra $C_{u}^{*}(G)$ and has other important applications [8, Chapter I].

The first quantum version of Wang's characterisation of Property $(\mathrm{T})$ was proven for discrete quantum groups (see [29, Remark 5.4]). Under the additional hypothesis of having low 
duals, Bekka-Valette and Kerr-Pichot theorems were proven for unimodular discrete quantum groups [18, Theorem 7.3, $7.6 \&$ 9.3]. Recall that for discrete quantum groups being unimodular is the same as being a Kac algebra and that Kac algebras form a class of locally compact quantum groups with trivial scaling group. The study of Property (T) on quantum groups progressed along these lines with the quantum versions of the theorems of Wang, Bekka-Valette and Kerr-Pichot generalised to quantum groups with trivial scaling groups in [10, Proposition $3.2 \&$ Theorem 3.6] and in [9, Theorem 4.7, 4.8, $4.9 \&$ 5.1].

Upon giving an affirmative answer to the Admissibility Conjecture for unimodular locally compact quantum groups (including those with non-trivial scaling groups), we proceed to prove a quantum version of Wang's theorem for them as well as generalised versions of the Bekka-Valette and the Kerr-Pichot theorems. In particular, we show that for unimodular quantum groups with non-trivial scaling automorphism group, the weakly mixing representations are dense in the set of representations on a separable Hilbert space if the quantum group does not have Property (T).

Acknowledgement. We are grateful to Adam Skalski for various mathematical discussions. All the authors would also like to thank Ami Viselter for various mathematical comments on an earlier version of the paper. The first author gratefully acknowledges the support of the Mathematical Research Unit at the University of Oulu, Finland, during the years 20152017 and partial support of the Simons Foundation grant 346300 and the Polish Government MNiSW 2015-2019 matching fund.

\section{NOTATION AND TERMiNOLOGY}

We collect a few facts from the theory of locally compact quantum groups, as developed in the papers [26, 27, 28], and we refer the reader to [25] for a summary of the main results in the theory. We will take the viewpoint that whenever we consider a locally compact quantum group, the symbol $\mathbb{G}$ denotes the underlying 'locally compact quantum space' of the quantum group. From this viewpoint, for a locally compact quantum group $\mathbb{G}$ the corresponding $\mathrm{C}^{*}$ algebra of 'continuous functions on $\mathbb{G}$ vanishing at infinity' will be denoted by $C_{0}(\mathbb{G})$. It is equipped with a coassociative comultiplication $\Delta: C_{0}(\mathbb{G}) \rightarrow M\left(C_{0}(\mathbb{G}) \otimes C_{0}(\mathbb{G})\right)$ and left and right Haar weights $\phi$ and $\psi[27$, Definition 4.1] (where we use the notation that $M(A)$ denotes the multiplier algebra of a $\mathrm{C}^{*}$-algebra $A$ ). An important aspect of the theory of locally compact quantum groups is a noncommutative Pontryagin duality theory, which in particular allows one to view both a locally compact group and its 'dual' as locally compact quantum groups [25, Subsection 6.2], [27, Section 8]. The dual of $\mathbb{G}$, which is again a locally compact quantum group, is denoted by $\widehat{\mathbb{G}}$. (For example if $\mathbb{G}=G$, a locally compact group, then $C_{0}(\widehat{\mathbb{G}})=C_{r}^{*}(G)$, the reduced group $C^{*}$-algebra of $G$.) As in the case of $\mathbb{G}, C_{0}(\widehat{\mathbb{G}})$ is equipped with a coassociative comultiplication $\widehat{\Delta}: C_{0}(\widehat{\mathbb{G}}) \rightarrow M\left(C_{0}(\widehat{\mathbb{G}}) \otimes C_{0}(\widehat{\mathbb{G}})\right)$ and left and right Haar weights $\widehat{\phi}$ and $\widehat{\psi}$. By the definition of the dual quantum group as given in [27, Definition 8.1], we may think of both the $\mathrm{C}^{*}$-algebras $C_{0}(\mathbb{G})$ and $C_{0}(\widehat{\mathbb{G}})$ as acting faithfully and non-degenerately on the Hilbert space $L^{2}(\mathbb{G}$ ) (obtained by applying the GNS construction to the left Haar weight $\phi$ ). A locally compact quantum group is said to be compact if $C_{0}(\mathbb{G})$ is unital. Compact quantum groups themselves have a very nice theory [47, 30].

The fundamental multiplicative unitary W $\in M\left(C_{0}(\mathbb{G}) \otimes C_{0}(\widehat{\mathbb{G}})\right.$ ) (called the Kac-Takesaki operator in the theory of Kac algebras [19], a precursor to the theory of locally compact 
quantum groups) implements the comultiplications as follows:

$$
\Delta(x)=\mathrm{W}^{*}(1 \otimes x) \mathrm{W}, \quad x \in C_{0}(\mathbb{G}),
$$

and

$$
\widehat{\Delta}(x)=\chi\left(\mathrm{W}(x \otimes 1) \mathrm{W}^{*}\right), \quad x \in C_{0}(\widehat{\mathbb{G}}),
$$

where $\chi: B\left(L^{2}(\mathbb{G}) \otimes L^{2}(\mathbb{G})\right) \rightarrow B\left(L^{2}(\mathbb{G}) \otimes L^{2}(\mathbb{G})\right)$ is the flip map [25, Definition 6.12 \& Subsection 6.2], [27, pp. 872-873, Definition 8.1].

The von Neumann algebra generated by $C_{0}(\mathbb{G})$ (respectively, by $\left.C_{0}(\widehat{\mathbb{G}})\right)$ in $B\left(L^{2}(\mathbb{G})\right.$ ) will be denoted by $L^{\infty}(\mathbb{G})$ (respectively, by $L^{\infty}(\widehat{\mathbb{G}})$ ). Then the preduals of $L^{\infty}(\mathbb{G})$ and $L^{\infty}(\widehat{\mathbb{G}})$ are denoted by $L^{1}(\mathbb{G})$ and $L^{1}(\widehat{\mathbb{G}})$, respectively. The above formulas imply that both the maps $\Delta$ and $\widehat{\Delta}$ can be lifted to normal $*$-homomorphisms on $L^{\infty}(\mathbb{G})$ and $L^{\infty}(\widehat{\mathbb{G}})$. The preadjoints of the normal maps $\Delta$ and $\widehat{\Delta}$ equip $L^{1}(\mathbb{G})$ and $L^{1}(\widehat{\mathbb{G}})$ with the structure of a completely contractive Banach algebra. The universal $\mathrm{C}^{*}$-algebra $C_{0}^{u}(\mathbb{G})$ associated with $\mathbb{G}$ is the universal $\mathrm{C}^{*}$-envelope of a distinguished Banach *-algebra $L_{\sharp}^{1}(\widehat{\mathbb{G}})$ (as an algebra, $\left.L_{\sharp}^{1}(\widehat{\mathbb{G}}) \subset L^{1}(\widehat{\mathbb{G}})\right)$. The $\mathrm{C}^{*}$-algebra $C_{0}^{u}(\mathbb{G})$ is equipped with a coassociative comultiplication denoted by $\Delta_{u}: C_{0}^{u}(\mathbb{G}) \rightarrow M\left(C_{0}^{u}(\mathbb{G}) \otimes C_{0}^{u}(\mathbb{G})\right)$ [26. Proposition 6.1]. Moreover, there exists a surjective $*$-homomorphism $\Lambda_{\mathbb{G}}: C_{0}^{u}(\mathbb{G}) \rightarrow C_{0}(\mathbb{G})$ called the reducing morphism, which intertwines the comultiplications: $\left(\Lambda_{\mathbb{G}} \otimes \Lambda_{\mathbb{G}}\right) \circ \Delta_{u}=\Delta \circ \Lambda_{\mathbb{G}}$. The comultiplication on $C_{0}^{u}(\widehat{\mathbb{G}})$ is denoted by $\widehat{\Delta}_{u}$. The dual space $C_{0}^{u}(\mathbb{G})^{*}$ is a completely contractive Banach algebra with respect to the convolution product

$$
\omega_{1} \star \omega_{2}=\left(\omega_{1} \otimes \omega_{2}\right) \circ \Delta_{u}, \quad \omega_{1}, \omega_{2} \in C_{0}^{u}(\mathbb{G})^{*} .
$$

As shown in [26, Corollary 4.3 and Proposition 5.2], the fundamental multiplicative unitary W admits a lift W $\in M\left(C_{0}(\mathbb{G}) \otimes C_{0}^{u}(\widehat{\mathbb{G}})\right)$ called the semi-universal bicharacter of $\mathbb{G}$. It is characterised by the following universal property: there is a one-to-one correspondence between

- unitary elements $U \in M\left(C_{0}(\mathbb{G}) \otimes B\right)$ such that $(\Delta \otimes \iota)(U)=U_{13} U_{23}$ (here $B$ is a $\mathrm{C}^{*}$-algebra)

- non-degenerate $*$-homomorphisms $\pi_{U}: C_{0}^{u}(\widehat{\mathbb{G}}) \rightarrow M(B)$ satisfying $\left(\iota \otimes \pi_{U}\right)(\mathrm{W})=U$.

There are two important maps associated with a locally compact quantum group $\mathbb{G}$ related to the inverse operation of a group. The antipode $S$ is a densely defined norm-closed map on $C_{0}(\mathbb{G})\left[27\right.$, Section 5]. It can be extended to a densely defined strictly closed map on $M\left(C_{0}(\mathbb{G})\right)$ 27. Remark 5.44]. The antipode has a universal counterpart $S_{u}$ which is a densely defined map on $C_{0}^{u}(\mathbb{G})\left[26\right.$, Section 9]. The unitary antipode $R: C_{0}(\mathbb{G}) \rightarrow C_{0}(\mathbb{G})$ is a $*$-antiautomorphism [27. Proposition 5.20] satisfying $(R \otimes R) \circ \Delta=\chi \circ \Delta \circ R$. Its universal counterpart $R_{u}$ is a *-antiautomorphism of $C_{0}^{u}(\mathbb{G})$ having similar properties as $R$ [26, Proposition 7.2]. The corresponding maps on the dual quantum group are denoted by $\widehat{S}, \widehat{S}_{u}, \widehat{R}$ and $\widehat{R}_{u}$. It is worthwhile to note that if $\mathbb{G}$ is a Kac algebra, then $S=R$ and $S_{u}=R_{u}$. In general, the antipode has a polar decomposition $S=R \circ \tau_{-i / 2}$ where $\tau_{i / 2}$ is defined by an analytic extension of the scaling automorphism group $\left(\tau_{t}\right)_{t \in \mathbb{R}}$, where each $\tau_{t}: C_{0}(\mathbb{G}) \rightarrow C_{0}(\mathbb{G})$ is a *automorphism. The scaling group is implemented by the modular operator $\widehat{\nabla}$ of the dual left Haar weight. (If $\mathbb{G}$ is a Kac algebra, $\widehat{\nabla}$ is affiliated to the center of $L^{\infty}(\mathbb{G})$ and consequently $\tau_{t}=\iota$ for every $t \in \mathbb{R}$.) The scaling group of the dual quantum group $\widehat{\mathbb{G}}$ is denoted by $\widehat{\tau}$. The scaling groups have their universal counterparts on the $\mathrm{C}^{*}$-algebras $C_{0}^{u}(\mathbb{G})$ and $C_{0}^{u}(\widehat{\mathbb{G}})$ and 
these are denoted by $\tau^{u}$ and $\widehat{\tau}^{u}$, respectively (see [26, Definition 4.1]). The universal antipode has a similar decomposition $S_{u}=R^{u} \circ \tau_{-i / 2}^{u}$.

The modular automorphism group associated to the left Haar weight $\phi$ on $\mathbb{G}$ is denoted by $\left(\sigma_{t}\right)_{t \in \mathbb{R}}$, and its universal counterpart by $\left(\sigma_{t}^{u}\right)_{t \in \mathbb{R}}$.

As shown in [26, Proposition 6.3] there exist counits $\epsilon_{u}: C_{0}^{u}(\mathbb{G}) \rightarrow \mathbb{C}$ and $\widehat{\epsilon}_{u}: C_{0}^{u}(\widehat{\mathbb{G}}) \rightarrow \mathbb{C}$, which are $*$-homomorphisms satisfying

$$
\left(\epsilon_{u} \otimes \iota\right)\left(\Delta_{u}(x)\right)=x=\left(\iota \otimes \epsilon_{u}\right)\left(\Delta_{u}(x)\right), \quad x \in C_{0}^{u}(\mathbb{G}),
$$

and

Moreover, $\left(\iota \otimes \widehat{\epsilon}_{u}\right)(\mathrm{W})=1$.

$$
\left(\widehat{\epsilon}_{u} \otimes \iota\right)\left(\widehat{\Delta}_{u}(x)\right)=x=\left(\iota \otimes \widehat{\epsilon}_{u}\right)\left(\widehat{\Delta}_{u}(x)\right), \quad x \in C_{0}^{u}(\widehat{\mathbb{G}}) .
$$

A representation of a locally compact quantum group $\mathbb{G}$ on a Hilbert space $H$ is an invertible element $U \in M\left(C_{0}(\mathbb{G}) \otimes \mathcal{K}(H)\right)$ such that

$$
(\Delta \otimes \iota)(U)=U_{13} U_{23}
$$

We are mostly interested in unitary representations in which case $U$ is further a unitary. Note that if $U \in L^{\infty}(\mathbb{G}) \otimes B(H)$ is a unitary that satisfies $(\mathbb{1})$, then $U \in M\left(C_{0}(\mathbb{G}) \otimes \mathcal{K}(H)\right)$. Indeed, (11) implies that

$$
U_{13}=\mathrm{W}_{12}^{*} U_{23} \mathrm{~W}_{12} U_{23}^{*} \in M\left(C_{0}(\mathbb{G}) \otimes \mathcal{K}\left(L^{2}(\mathbb{G})\right) \otimes \mathcal{K}(H)\right)
$$

as $\mathrm{W} \in M\left(C_{0}(\mathbb{G}) \otimes \mathcal{K}\left(L^{2}(\mathbb{G})\right)\right)$, and the claim follows.

The trivial representation $1 \otimes 1 \in M\left(C_{0}(\mathbb{G}) \otimes \mathbb{C}\right)$ is denoted by 1 . Two representations $U$ and $V$ are similar if there is an invertible $a \in B\left(H_{V}, H_{U}\right)$ such that $V=\left(1 \otimes a^{-1}\right) U(1 \otimes a)$ (where $B\left(H_{V}, H_{U}\right)$ denotes the set of bounded linear maps from $H_{V}$ to $\left.H_{U}\right)$. If $a$ is further an unitary map $U$ and $V$ are said to be (unitarily) equivalent. Given a representation $U \in$ $M\left(C_{0}(\mathbb{G}) \otimes \mathcal{K}(H)\right)$, its contragradient representation is

$$
U^{c}=(R \otimes \top) U \in M\left(C_{0}(\mathbb{G}) \otimes \mathcal{K}(\bar{H})\right)
$$

where $R$ is the unitary antipode, $\top(x) \bar{\xi}=\overline{x^{*} \xi}$ for $x \in B(H)$ and $\xi \in H$ and $\bar{H}$ is the dual Hilbert space of $H$.

If $U \in M\left(C_{0}(\mathbb{G}) \otimes \mathcal{K}(H)\right)$ and $V \in M\left(C_{0}(\mathbb{G}) \otimes \mathcal{K}(K)\right)$ are representations of $\mathbb{G}$, their tensor product is

$$
U \odot V=U_{12} V_{13} \in M\left(C_{0}(\mathbb{G}) \otimes \mathcal{K}(H \otimes K)\right) .
$$

As noted above, every unitary representation $U$ of $\mathbb{G}$ is associated with a representation $\pi$ of the $C^{*}$-algebra $C_{0}^{u}(\widehat{\mathbb{G}})$ via $U=(\iota \otimes \pi) \mathrm{W}$, and vice versa. In particular, the trivial representation is associated with the counit $\widehat{\epsilon}_{u}$. If $\pi_{U}$ and $\pi_{V}$ are the representations of $C_{0}^{u}(\widehat{\mathbb{G}})$ associated with $U$ and $V$, respectively, then the representation $\left(\pi_{U} \otimes \pi_{V}\right) \circ \chi \circ \widehat{\Delta}_{u}$ is associated with $U \odot V$.

\section{A CHARACTERISATION OF ADMisSiBlE FINITE-DIMENSIONAL UNITARY REPRESENTATIONS}

Let $U \in M\left(C_{0}(\mathbb{G}) \otimes M_{n}(\mathbb{C})\right)$ be a finite-dimensional unitary representation of a locally compact quantum group $\mathbb{G}$. Choosing the standard basis for $\mathbb{C}^{n}$ we write $U=\left(U_{i j}\right)_{i, j=1}^{n}$, where $U_{i j} \in M\left(C_{0}(\mathbb{G})\right)$ for $i, j=1,2, \ldots, n$ are the matrix coefficients of $U$, and we have $\Delta\left(U_{i j}\right)=\sum_{k=1}^{n} U_{i k} \otimes U_{k j}$ for $i, j=1,2, \ldots, n$. 
Definition 3.1. A finite-dimensional unitary representation $U=\left(U_{i j}\right)_{i, j=1}^{n}$ of a locally compact quantum group $\mathbb{G}$ is called admissible if $U^{t}:=\left(U_{j i}\right)_{i, j=1}^{n}$ is invertible in the $\mathrm{C}^{*}$-algebra $M\left(C_{0}(\mathbb{G}) \otimes M_{n}(\mathbb{C})\right)$.

Admissible finite-dimensional representations of locally compact quantum groups first appeared in the work of Soltan [39], who introduced the quantum Bohr compactification of a locally compact quantum group. Daws [15] studied further the quantum Bohr compactification as well as questions related to admissibility. It was conjectured (see [15, Conjecture 7.2]) that every finite-dimensional unitary representation of a locally compact quantum group is admissible. Note that this conjecture is already false if we replace quantum group by quantum semigroup: a counterexample due to Woronowicz is given in [45, Example 4.1].

From the results in $[49]$ it follows that if $U \in M\left(C_{0}(\mathbb{G}) \otimes M_{n}(\mathbb{C})\right)$ is an admissible finitedimensional unitary representation, then the $\mathrm{C}^{*}$-algebra generated by the matrix coefficients of $U$ in $M\left(C_{0}(\mathbb{G})\right)$ gives rise to a compact matrix quantum group. It follows that a finitedimensional unitary representation of a locally compact quantum group is admissible if and only if it factors, in this sense, through a compact quantum group (as finite-dimensional representations of compact quantum groups are admissible). It is worthwhile to note that the use of $C_{0}(\mathbb{G})$ above is purely a matter of convenience: we can do similar considerations for $C_{0}^{u}(\mathbb{G})$ as well.

The linear span of all matrix coefficients of admissible unitary representations of $\mathbb{G}$ is denoted by $\mathcal{A P}(\mathbb{G})$. Note that $\mathcal{A P}(\mathbb{G})$ is a Hopf $*$-algebra. Its norm closure in $M\left(C_{0}(\mathbb{G})\right)$ is denoted by $A P(\mathbb{G})$. It may be that $A P(\mathbb{G})$ is not the universal $\mathrm{C}^{*}$-completion of $\mathcal{A P}(\mathbb{G})$. The compact quantum group associated with $\mathcal{A P}(\mathbb{G})$ is the quantum Bohr compactification of $\mathbb{G}$ and is denoted by $\mathfrak{b} \mathbb{G}$. See [39, 15] for more details.

Next we characterise the admissibility of a finite-dimensional unitary representation in terms of the scaling group.

Proposition 3.2. Let $\pi: C_{0}^{u}(\widehat{\mathbb{G}}) \rightarrow M_{n}(\mathbb{C})$ be a non-degenerate *-homomorphism, $U=$ $(\iota \otimes \pi)(\mathrm{W})$ and $L_{U}=\operatorname{span}\left\{U_{i j}: i, j=1,2, \ldots, n\right\}$. Then the following statements are equivalent:

(i) The representation $U \in M\left(C_{0}(\mathbb{G}) \otimes M_{n}(\mathbb{C})\right)$ is admissible.

(ii) The vector space $L_{U}$ is invariant under the scaling group $\left(\tau_{t}\right)$.

(iii) There exists a strongly continuous one-parameter automorphism group $\left(\alpha_{t}\right)$ on $M_{n}(\mathbb{C})$ such that

$$
\pi \circ \widehat{\tau}_{t}^{u}=\alpha_{t} \circ \pi \quad \text { for every } t \in \mathbb{R} .
$$

Proof. (i) $\Longrightarrow$ (iii): Since $U=\left(U_{i j}\right)$ is admissible, $U_{i j} \in \mathcal{A P}(\mathbb{G})$ for all $i, j=1,2, \ldots, n$. Let $\Theta: C^{u}(\mathfrak{b} \mathbb{G}) \rightarrow A P(\mathbb{G}) \subset M\left(C_{0}(\mathbb{G})\right)$ be the canonical quantum group morphism from the universal $C^{*}$-completion of $\mathcal{A P}(\mathbb{G})$ onto the closure of $\mathcal{A P}(\mathbb{G})$. Let $\widehat{\Theta}: C_{0}^{u}(\widehat{\mathbb{G}}) \rightarrow M\left(C_{0}^{u}(\widehat{\mathfrak{b} \mathbb{G}})\right)$ be the dual morphism so that $(\iota \otimes \widehat{\Theta}) \mathbb{W}=(\Theta \otimes \iota) \mathbb{W}_{\mathfrak{b} \mathbb{G}}$ (see [32, Corollary 4.3]). Since $\widehat{\mathfrak{b} \mathbb{G}}$ is a discrete quantum group, we can drop $u$ from the notation and write $c_{0}(\widehat{\mathfrak{b} \mathbb{G}})$ for notational convenience.

Let $\widetilde{U} \in M\left(C^{u}(\mathfrak{b} \mathbb{G}) \otimes M_{n}(\mathbb{C})\right)$ be the lift of the representation $U$, i.e. $(\Theta \otimes \iota) \widetilde{U}=U$. Then there is a non-degenerate $*$-homomorphism $\phi: c_{0}(\widehat{\mathfrak{b} \mathbb{G}}) \rightarrow M_{n}(\mathbb{C})$ such that $\widetilde{U}=(\iota \otimes \phi) \mathbb{W}_{\mathfrak{b} \mathbb{G}}$. We have

which implies that $\pi=\phi \circ \widehat{\Theta}$.

$$
(\iota \otimes \phi \circ \widehat{\Theta}) \mathrm{W}=(\Theta \otimes \phi) \mathbb{W}_{\mathfrak{b} \mathbb{G}}=U=(\iota \otimes \pi) \mathrm{W},
$$


There is an unbounded strictly positive operator $K$ affiliated to the von Neumann algebra $\ell^{\infty}(\widehat{\mathfrak{b} G})$ such that $K$ implements the scaling group $\left(\tau_{t}^{\prime}\right)$ of $\widehat{\mathfrak{b} \mathbb{G}}$ in the sense that $\tau_{t}^{\prime}(x)=$ $K^{-2 i t} x K^{2 i t}$ for every $x \in \ell^{\infty}(\widehat{\mathfrak{b} \mathbb{G}})$ and $t \in \mathbb{R}\left[13\right.$, Proposition 4.3]. Note that $K^{2 i t} \in \ell^{\infty}(\widehat{\mathfrak{b}} \widehat{\mathbb{G}})$ for all $t \in \mathbb{R}$.

Now for $x \in C_{0}^{u}(\widehat{\mathbb{G}})$ and $t \in \mathbb{R}$,

$$
\pi\left(\widehat{\tau}_{t}^{u}(x)\right)=\phi\left(\widehat{\Theta}\left(\widehat{\tau}_{t}^{u}(x)\right)\right)=\phi\left(\tau_{t}^{\prime}(\widehat{\Theta}(x))\right)
$$

since $\widehat{\Theta}$ intertwines the scaling groups as a morphism of quantum groups (by [26, Remark 12.1]). Continuing the calculation, we have

$$
\pi\left(\widehat{\tau}_{t}^{u}(x)\right)=\phi\left(K^{-2 i t} \widehat{\Theta}(x) K^{2 i t}\right)=\phi\left(K^{-2 i t}\right) \pi(x) \phi\left(K^{2 i t}\right) .
$$

Defining $\alpha_{t}(A)=\phi\left(K^{-2 i t}\right) A \phi\left(K^{2 i t}\right)$ for $t \in \mathbb{R}$ and $A \in M_{n}(\mathbb{C})$ yields the desired result.

(iii) $\Longrightarrow$ (ii): From the version of [26, Proposition 9.1] for $C_{0}^{u}(\widehat{\mathbb{G}})$, we have $\left(\tau_{t} \otimes \iota\right)(\mathrm{W})=$ $\left(\iota \otimes \widehat{\tau}_{t}^{u}\right) \mathrm{W}$ for all $t \in \mathbb{R}$. Then

$$
\begin{aligned}
\left(\tau_{t} \otimes \iota\right)(U) & =\left(\tau_{t} \otimes \iota\right) \circ(\iota \otimes \pi)(\mathrm{W})=\left(\iota \otimes \pi \circ \widehat{\tau}_{t}^{u}\right)(\mathrm{W}) \\
& =\left(\iota \otimes \alpha_{t} \circ \pi\right)(\mathrm{W})=\left(\iota \otimes \alpha_{t}\right)(U) .
\end{aligned}
$$

It follows that $L_{U}$ is invariant under the scaling group $\left(\tau_{t}\right)$

(ii) $\Longrightarrow$ (i): Let $\tau_{z}$ denote the analytic extension of $\left(\tau_{t}\right)_{t \in \mathbb{R}}$ at $z \in \mathbb{C}$ in the $\sigma$-weak topology on $L^{\infty}(\mathbb{G})$. For $x \in L_{U}$ define in a standard way the smear of $x$

$$
x_{n}=\frac{n}{\sqrt{\pi}} \int_{\mathbb{R}} e^{-n^{2} t^{2}} \tau_{t}(x) d t,
$$

where the integral converges in the $\sigma$-weak topology. Each $x_{n}$ is analytic for $\left(\tau_{t}\right)$. As $L_{U}$ is invariant under the scaling group and is finite-dimensional, it follows that $x_{n} \in L_{U}$ for each $n$. It follows that $L_{U} \subseteq D\left(\tau_{z}\right)$ for all $z$. In particular, $L_{U} \subset D\left(\tau_{\frac{i}{2}}\right)=D\left(S^{-1}\right)$, due to the polar decomposition of $S^{-1}$. It then follows that $U_{i j}^{*} \in D(S)$ for all $i, j=1,2, \ldots, n$. By [15, Proposition 3.11], $U$ is admissible.

Remark 3.3. It follows from Proposition 3.2 that for quantum groups with trivial scaling automorphism group, all finite-dimensional unitary representations are admissible. In particular the Admissibility Conjecture holds for Kac algebras, as also noted in [15].

Remark 3.4. For a general locally compact quantum group, it follows that admissible representations of $\mathbb{G}$ correspond to those finite-dimensional representations of $C_{0}^{u}(\widehat{\mathbb{G}})$ which are covariant with respect to the scaling action of $\mathbb{R}$ on $C_{0}^{u}(\widehat{\mathbb{G}})$.

Remark 3.5. Let $U \in M\left(C_{0}(\mathbb{G}) \otimes M_{n}(\mathbb{C})\right)$ be admissible. Then $U$ is a corepresentation of the compact quantum group $A P(\mathbb{G})$. As the inclusion $A P(\mathbb{G}) \rightarrow M\left(C_{0}(\mathbb{G})\right)$ intertwines $R$, we see that $U^{c}$ may also be considered as a corepresentation of the compact quantum group $A P(\mathbb{G})$. Combining [33, Definition 1.3.8, Definition 1.4.5 and equation (1.7.1)] we can conclude that $\bar{U}=\left(U_{i j}^{*}\right)_{i, j=1}^{n}$ is equivalent to $U^{c}$, as corepresentations of $A P(\mathbb{G})$, and hence also of $C_{0}(\mathbb{G})$ (it is worthwhile to point out that in [33, $U^{c}$ and $\bar{U}$ are what we call $\bar{U}$ and $U^{c}$ respectively in this article). 


\section{Examples of locally Compact quantum groups With admissible REPRESENTATIONS}

It is an open question whether all finite-dimensional unitary representations of a locally compact quantum group are admissible. In this subsection we give examples of locally compact quantum groups for which the Admissibility Conjecture is true.

4.1. Quantum groups arising from a bicrossed product construction. We recall some facts about the bicrossed product construction of a matched pair of quantum groups and refer to [42] for details. Define a normal $*$-homomorphism $\tau$ as follows:

$$
\tau: L^{\infty}(\mathbb{G}) \bar{\otimes} L^{\infty}(\mathbb{R}) \rightarrow L^{\infty}(\mathbb{G}) \bar{\otimes} L^{\infty}(\mathbb{R}), \quad \tau(f)(t)=\tau_{t}(f(t)) \quad\left(f \in L^{\infty}(\mathbb{G}) \bar{\otimes} L^{\infty}(\mathbb{R}), t \in \mathbb{R}\right)
$$

where we have identified $L^{\infty}(\mathbb{G}) \bar{\otimes} L^{\infty}(\mathbb{R})$ with $L^{\infty}\left(\mathbb{R}, L^{\infty}(\mathbb{G})\right)$. Then the map $\tau$ is a matching between the locally compact quantum groups $\mathbb{G}$ and $\mathbb{R}$ with trivial cocycles ( $\mathscr{U}=1$ and $\mathscr{V}=1$ ), in the sense of [42, Definition 2.1].

In the notation of [42, Definition 2.1], we define a left action $\alpha: L^{\infty}(\mathbb{R}) \rightarrow L^{\infty}(\mathbb{G}) \bar{\otimes} L^{\infty}(\mathbb{R})$ and a right action $\beta: L^{\infty}(\mathbb{G}) \rightarrow L^{\infty}(\mathbb{G}) \bar{\otimes} L^{\infty}(\mathbb{R})$ by the formulas

$$
\alpha(f)=\tau(1 \otimes f)=1 \otimes f, \quad f \in L^{\infty}(\mathbb{R}),
$$

and

$$
\beta(x)(t)=\tau(x \otimes 1)(t)=\tau_{t}(x), \quad t \in \mathbb{R}, x \in L^{\infty}(\mathbb{G}) .
$$

Consider the crossed products $M:=\mathbb{G}_{\alpha} \ltimes L^{\infty}(\mathbb{R})$ and $\widehat{M}:=L^{\infty}(\mathbb{G}) \rtimes_{\beta} \mathbb{R}$. It follows from the discussion in [42, Subsection 2.2] that $M$ is a locally compact quantum group in the reduced form and $\widehat{M}$ is the reduced dual. Note that since the left action $\alpha$ is trivial, $M=$ $L^{\infty}(\widehat{\mathbb{G}}) \bar{\otimes} L^{\infty}(\mathbb{R})$.

Denote the quantum group underlying $\widehat{M}$ by $\mathbb{G} \rtimes_{\beta} \mathbb{R}$. The left multiplicative unitary of $\mathbb{G} \rtimes_{\beta} \mathbb{R}$ is

$$
\mathrm{W}=\mathrm{W}_{24}^{\mathbb{R}}\left((\iota \otimes \beta)\left(\widehat{\mathrm{W}}^{\mathbb{G}}\right)\right)_{134},
$$

where the leg numbering refers to the underlying Hilbert space $L^{2}(\mathbb{G}) \otimes L^{2}(\mathbb{R}) \otimes L^{2}(\mathbb{G}) \otimes L^{2}(\mathbb{R})$ (see [4, p. 141]). It follows from the above formula that $C_{0}\left(\mathbb{G} \rtimes_{\beta} \mathbb{R}\right)=C_{0}(\mathbb{G}) \rtimes_{\beta} \mathbb{R}$. We will show a similar characterisation of $C_{0}^{u}\left(\mathbb{G} \rtimes_{\beta} \mathbb{R}\right)$. Consider the action

$$
\beta^{u}: C_{0}^{u}(\mathbb{G}) \rightarrow M\left(C_{0}^{u}(\mathbb{G}) \otimes C_{0}(\mathbb{R})\right), \quad \beta^{u}(x)(t)=\tau_{t}^{u}(x), \quad x \in C_{0}^{u}(\mathbb{G}), t \in \mathbb{R} .
$$

Lemma 4.1. $C_{0}^{u}\left(\mathbb{G} \rtimes_{\beta} \mathbb{R}\right) \cong C_{0}^{u}(\mathbb{G}) \rtimes_{\beta^{u}} \mathbb{R}$.

Proof. Denote the comultiplication of $\mathbb{G} \ltimes_{\alpha} \mathbb{R}$ by $\Delta$. We recall the following formula from [4, p. 141]:

$$
(\iota \otimes \Delta)\left(\mathrm{W}^{\mathbb{G}} \otimes 1_{L^{\infty}(\mathbb{R})}\right)=\mathrm{W}_{14}^{\mathbb{G}}((\iota \otimes \alpha) \circ \beta \otimes \iota)\left(\mathrm{W}^{\mathbb{G}}\right)_{1452},
$$

where the leg numbering is done with respect to the Hilbert space $L^{2}(\mathbb{G}) \otimes L^{2}(\mathbb{G}) \otimes L^{2}(\mathbb{R}) \otimes$ $L^{2}(\mathbb{G}) \otimes L^{2}(\mathbb{R})$. Applying the definition of $\alpha$, we obtain

$$
(\iota \otimes \Delta)\left(\mathrm{W}^{\mathbb{G}} \otimes 1_{L^{\infty}(\mathbb{R})}\right)=\mathrm{W}_{14}^{\mathbb{G}}\left((\iota \otimes \chi) \circ(\beta \otimes \iota)\left(\mathrm{W}^{\mathbb{G}}\right)\right)_{125} .
$$

where $\chi: L^{\infty}(\mathbb{R}) \bar{\otimes} L^{\infty}(\widehat{\mathbb{G}}) \rightarrow L^{\infty}(\widehat{\mathbb{G}}) \bar{\otimes} L^{\infty}(\mathbb{R})$ is the flip map. Write $U=(\iota \otimes \chi) \circ\left(\beta^{u} \otimes \iota\right)\left(\mathbb{W}^{\mathbb{G}}\right)$. Then (2) says that

$$
\left(\Lambda_{\mathbb{G}} \otimes \iota \otimes \iota \otimes \iota \otimes \iota\right)\left((\iota \otimes \Delta)\left(\mathbb{W}^{\mathbb{G}} \otimes 1_{L^{\infty}(\mathbb{R})}\right)\right)=\left(\Lambda_{\mathbb{G}} \otimes \iota \otimes \iota \otimes \iota \otimes \iota\right)\left(\mathbb{W}_{14}^{\mathbb{G}} U_{125}\right) .
$$


where $\Lambda_{\mathbb{G}}: C_{0}^{u}(\mathbb{G}) \rightarrow C_{0}(\mathbb{G})$ is the reducing morphism. We would like to apply [26, Result 6.1] (or a version of it for $\mathbb{G}$ ) to deduce that in fact

$$
(\iota \otimes \Delta)\left(\mathbb{W}^{\mathbb{G}} \otimes 1_{L^{\infty}(\mathbb{R})}\right)=\mathbb{W}_{14}^{\mathbb{G}} U_{125}
$$

To this end, we need to check that both sides of the preceding equation define corepresentations of $C_{0}^{u}(\mathbb{G})$.

Since $\left(\tau_{t}^{u} \otimes \tau_{t}^{u}\right) \circ \Delta_{u}=\Delta_{u} \circ \tau_{t}^{u}$ for all $t \in \mathbb{R}$, it follows that $U \in M\left(C_{0}^{u}(\mathbb{G}) \otimes \mathcal{K}\left(L^{2}(\mathbb{G}) \otimes L^{2}(\mathbb{R})\right)\right.$ is a corepresentation of $C_{0}^{u}(\mathbb{G})$. Therefore, $\mathbb{W}^{\mathbb{G}} \odot U$ is a corepresentation and hence also

$$
\left((\iota \otimes \chi \otimes \iota)\left(\mathbb{W}^{\mathbb{G}} \odot U\right)\right)_{1245}
$$

where $\chi$ is the appropriate flip map. It follows that the right-hand side of (3) is a corepresentation, and the left-hand side clearly is as well.

Suppose that $X \in M\left(C_{0}\left(\mathbb{G} \ltimes_{\alpha} \mathbb{R}\right) \otimes \mathcal{K}(H)\right)$ is a unitary representation of $\mathbb{G} \ltimes_{\alpha} \mathbb{R}$. By 4 , Proposition 4.1] there are unitary representations $z \in M\left(C_{0}(\widehat{\mathbb{G}}) \otimes \mathcal{K}(H)\right)$ and $y \in M\left(C_{0}(\mathbb{R}) \otimes\right.$ $\mathcal{K}(H))$ such that $X=(\alpha \otimes \iota)(y) z_{13}$, where the leg numbering is done with respect to the Hilbert space $L^{2}(\mathbb{G}) \otimes L^{2}(\mathbb{R}) \otimes H$. (The unitarity of the representations is implicit in the proof of [4, Proposition 4.1].) The equation after equation (4.2) in page 146 of [4] says that

$$
(\Delta \otimes \iota)\left(z_{13}\right) z_{35}^{*}=(\alpha \otimes \iota)\left(y^{*}\right)_{345} z_{15}(\alpha \otimes \iota)(y)_{345},
$$

where the leg numbering is done with respect to the Hilbert space

$$
L^{2}(\mathbb{G}) \otimes L^{2}(\mathbb{R}) \otimes L^{2}(\mathbb{G}) \otimes L^{2}(\mathbb{R}) \otimes H .
$$

Applying the fact that $\alpha(f)=1_{L^{\infty}(\mathbb{G})} \otimes f$ for all $f \in L^{\infty}(\mathbb{R})$, it follows that the above equation can be reduced to:

$$
(\Delta \otimes \iota)\left(z_{13}\right) z_{35}^{*}=y_{45}^{*} z_{15} y_{45} .
$$

Let $\pi: C_{0}^{u}(\mathbb{G}) \rightarrow B(H)$ be the non-degenerate $\mathrm{C}^{*}$-representation associated with $z$, so that we have $(\iota \otimes \pi)\left(\widehat{\mathrm{W}^{\mathbb{G}}}\right)=z$. Then it is easy to check that

$$
(\Delta \otimes \iota)\left(z_{13}\right)=(\iota \otimes \iota \otimes \iota \otimes \iota \otimes \pi)\left(\sigma\left((\iota \otimes \Delta)\left(\mathbb{W}^{\mathbb{G}^{*}} \otimes 1_{L^{\infty}(\mathbb{R})}\right)\right)\right)
$$

where

$\sigma: M\left(C_{0}^{u}(\mathbb{G}) \otimes C_{0}(\widehat{\mathbb{G}}) \otimes C_{0}(\mathbb{R}) \otimes C_{0}(\widehat{\mathbb{G}}) \otimes C_{0}(\mathbb{R})\right) \rightarrow M\left(C_{0}(\widehat{\mathbb{G}}) \otimes C_{0}(\mathbb{R}) \otimes C_{0}(\widehat{\mathbb{G}}) \otimes C_{0}(\mathbb{R}) \otimes C_{0}^{u}(\mathbb{G})\right)$ permutes the coordinates according to the permutation (15432).

Then, applying (3) and (4), we get

$$
(\iota \otimes \iota \otimes \iota \otimes \iota \otimes \pi)\left(\left((\iota \otimes \chi) \circ\left(\iota \otimes \beta^{u}\right)\left(\widehat{\mathrm{W}^{\mathbb{G}}}\right)\right)_{145}\right)=y_{45}^{*} z_{15} y_{45},
$$

where $\chi: M\left(C_{0}^{u}(\mathbb{G}) \otimes C_{0}(\mathbb{R})\right) \rightarrow M\left(C_{0}(\mathbb{R}) \otimes C_{0}^{u}(\mathbb{G})\right)$ is the usual flip (we are also using the fact that $\left.\widehat{\mathbb{W}^{\mathbb{G}}}=\Sigma\left(\mathbb{W}^{\mathbb{G}}\right)^{*} \Sigma\right)$.

Letting $y_{t}:=y(t) \in B(H)$ for $t \in \mathbb{R}$, it follows from the above equation that

$$
\pi\left(\tau_{t}^{u}(x)\right)=y_{t}^{*} \pi(x) y_{t}, \quad t \in \mathbb{R}, x \in C_{0}^{u}(\mathbb{G}) .
$$

Therefore, $\pi: C_{0}^{u}(\mathbb{G}) \rightarrow B(H)$ is a covariant $\mathrm{C}^{*}$-representation and hence lifts to a representation $\tilde{\pi}$ of the crossed product $\mathrm{C}^{*}$-algebra $C_{0}^{u}(\mathbb{G}) \rtimes_{\beta^{u}} \mathbb{R}$. Put

$$
\mathrm{W}:=\mathrm{W}_{24}^{\mathbb{R}}\left(\left(\iota \otimes \beta^{u}\right)\left(\widehat{\mathrm{W}^{\mathbb{G}}}\right)\right)_{134}
$$

The above argument shows that $X=(\iota \otimes \tilde{\pi})(\mathrm{W})$, so that $\mathrm{W}$ is a maximal corepresentation of $\left(C_{0}\left(\mathbb{G} \ltimes_{\alpha} \mathbb{R}\right), \Delta\right)$ in the sense of [40, Definition 23]. Therefore, the right leg of W generates 
the universal $\mathrm{C}^{*}$-algebra $C_{0}^{u}\left(\mathbb{G} \rtimes_{\beta} \mathbb{R}\right)$, but this is precisely $C_{0}^{u}(\mathbb{G}) \rtimes_{\beta^{u}} \mathbb{R}$, which is what we wanted to prove.

In our case, $\alpha$ being trivial, the left Haar weight of $\mathbb{G} \ltimes_{\alpha} \mathbb{R}$ is $\widehat{\phi} \otimes \psi$ where $\widehat{\phi}$ is the left Haar weight of $\widehat{\mathbb{G}}$ and $\psi$ the Haar weight of $\mathbb{R}$ (see [4, p. 141]). Hence, the modular operator of the left Haar weight of $\mathbb{G} \ltimes_{\alpha} \mathbb{R}$ is $\widehat{\nabla} \otimes I_{L^{2}(\mathbb{R})}$, where $\widehat{\nabla}$ is the modular operator of the left Haar weight of $\widehat{\phi}$. It follows that the scaling group of $\mathbb{G} \rtimes_{\beta} \mathbb{R}$ is given by $\widetilde{\tau}_{t}=\tau_{t} \otimes \iota_{B\left(L^{2}(\mathbb{R})\right)}$, $t \in \mathbb{R}$, and so the scaling group on $C_{0}^{u}\left(\mathbb{G} \rtimes_{\beta} \mathbb{R}\right)$ is given by $\widetilde{\tau}_{t}^{u}=\tau_{t}^{u} \otimes \iota_{B\left(L^{2}(\mathbb{R})\right)}, t \in \mathbb{R}$.

By the general theory of crossed products, the covariant representations of $C_{0}^{u}(\mathbb{G})$ with respect to $\left(\tau_{t}^{u}\right)$ correspond to the non-degenerate representations of $C_{0}^{u}(\mathbb{G}) \rtimes_{\beta^{u}} \mathbb{R}$ (note that $\mathbb{R}$ is amenable so the reduced and the full crossed products coincide). Now suppose that $\rho$ is a non-degenerate representation of $C_{0}^{u}(\mathbb{G}) \rtimes_{\beta^{u}} \mathbb{R}$. Then $\pi:=\rho \circ \beta^{u}$ is a covariant representation of $C_{0}^{u}(\mathbb{G})$ and the associated one-parameter unitary group is given by $U_{t}=\rho\left(1 \otimes \lambda_{t}\right)$ where $\lambda_{t} \in M\left(C^{*}(\mathbb{R})\right)$ is the left translation by $t \in \mathbb{R}$.

It is easy to see that $\widetilde{\tau}_{t}^{u} \circ \beta^{u}=\beta^{u} \circ \tau_{t}^{u}$ for all $t \in \mathbb{R}$. Now for every $f \in C_{c}(\mathbb{R})$ (the space of compactly supported continuous functions on $\mathbb{R})$ and $x \in C_{0}^{u}(\mathbb{G})$, we have

$$
\begin{aligned}
\rho\left(\widetilde{\tau}_{t}^{u}\left(\beta^{u}(x)(1 \otimes f)\right)\right) & =\rho\left(\beta^{u}\left(\tau_{t}^{u}(x)\right)(1 \otimes f)\right)=\pi\left(\tau_{t}^{u}(x)\right) \int_{\mathbb{R}} f(s) U_{s} d s \\
& =U_{t}^{*}\left(\pi(x) \int_{\mathbb{R}} f(s) U_{s} d s\right) U_{t}=U_{t}^{*}\left(\rho\left(\beta^{u}(x)(1 \otimes f)\right)\right) U_{t} .
\end{aligned}
$$

The norm-density of elements of the form $\beta^{u}(x)(1 \otimes f)$ in $C_{0}^{u}(\mathbb{G}) \rtimes_{\beta} \mathbb{R}$ implies that $\rho\left(\widetilde{\tau}_{t}^{u}(X)\right)=$ $U_{t}^{*}(\rho(X)) U_{t}$ for all $X \in C_{0}^{u}(\mathbb{G}) \rtimes_{\beta} \mathbb{R}$ and $t \in \mathbb{R}$. In particular, it follows from Proposition 3.2, that all finite-dimensional unitary representation of the quantum group $\mathbb{G} \ltimes_{\alpha} \mathbb{R}=\widehat{\mathbb{G} \rtimes_{\beta} \mathbb{R}}$ are admissible. We summarise these observations in the following theorem.

Theorem 4.2. Let $\mathbb{G}$ be a locally compact quantum group. Let $\left(\tau_{t}\right)_{t \in \mathbb{R}}$ be the scaling group and consider the matching

$$
\tau: L^{\infty}(\mathbb{G}) \bar{\otimes} L^{\infty}(\mathbb{R}) \rightarrow L^{\infty}(\mathbb{G}) \bar{\otimes} L^{\infty}(\mathbb{R}), \quad \tau(f)(t)=\tau_{t}(f(t)) \quad\left(f \in L^{\infty}(\mathbb{G}) \bar{\otimes} L^{\infty}(\mathbb{R}) \mathrm{n}\right. \text { of }
$$


Since $\mathbb{G}$ is unimodular, the universal modular automorphism groups of the right and left invariant weights are the same. It then follows from [26, Proposition 9.2] that

$$
\Delta_{u} \circ \sigma_{t}^{u}=\left(\tau_{t}^{u} \otimes \sigma_{t}^{u}\right) \circ \Delta_{u}
$$

and

$$
\Delta_{u} \circ \sigma_{t}^{u}=\left(\sigma_{t}^{u} \otimes \tau_{-t}^{u}\right) \circ \Delta_{u}
$$

for every $t \in \mathbb{R}$. Applying these to the matrix coefficient of $V$, we obtain

$$
\Delta_{u}\left(\sigma_{t}^{u}\left(V_{i j}\right)\right)=\sum_{k=1}^{n} \tau_{t}^{u}\left(V_{i k}\right) \otimes \sigma_{t}^{u}\left(V_{k j}\right)
$$

and

$$
\Delta_{u}\left(\sigma_{t}^{u}\left(V_{i j}\right)\right)=\sum_{k=1}^{n} \sigma_{t}^{u}\left(V_{i k}\right) \otimes \tau_{-t}^{u}\left(V_{k j}\right) .
$$

Then applying the counit of $C_{0}^{u}(\mathbb{G})$ to the above identities, it follows that $\tau_{-t}^{u}\left(\sigma_{t}^{u}\left(L_{V}\right)\right) \subset L_{V}$ and $\tau_{t}^{u}\left(\sigma_{t}^{u}\left(L_{V}\right)\right) \subset L_{V}$ for all $t \in \mathbb{R}$. This implies that for $X \in L_{V}$,

$$
\tau_{2 t}^{u}(X)=\left(\tau_{t}^{u} \circ \sigma_{-t}^{u}\right) \circ\left(\sigma_{t}^{u} \circ \tau_{t}^{u}\right)(X) \subset L_{V} .
$$

Therefore, $\tau_{t}^{u}\left(L_{V}\right) \subset L_{V}$ for all $t \in \mathbb{R}$.

Let $\Lambda_{\mathbb{G}}: C_{0}^{u}(\mathbb{G}) \rightarrow C_{0}(\mathbb{G})$ be the reducing morphism. Then $\tau_{t} \circ \Lambda_{\mathbb{G}}=\Lambda_{\mathbb{G}} \circ \tau_{t}^{u}$ for all $t \in \mathbb{R}$ (see [26, Section 4]). Write $U=\left(U_{i j}\right)$, and note that $\Lambda_{\mathbb{G}}\left(V_{i j}\right)=U_{i j}$. Since $L_{V}$ is $\tau_{t}^{u}$-invariant, it follows that $L_{U}$ is $\tau_{t}$-invariant. By Proposition [3.2, $U$ is admissible.

A class of examples of unimodular quantum groups with non-trivial scaling groups is given by Drinfeld's quantum doubles. Given a matched pair of locally compact quantum groups $\mathbb{G}$ and $\mathbb{H}$, the von Neumann algebra $L^{\infty}(\mathbb{G}) \bar{\otimes} L^{\infty}(\mathbb{H})$ can be given the structure of a locally compact quantum group, called the double crossed product of $\mathbb{G}$ and $\mathbb{H}[4$, Section $3 \&$ Theorem 5.3]. If $\mathbb{H}=\widehat{\mathbb{G}}$, then a matching

$$
m: L^{\infty}(\mathbb{G}) \bar{\otimes} L^{\infty}(\widehat{\mathbb{G}}) \rightarrow L^{\infty}(\mathbb{G}) \bar{\otimes} L^{\infty}(\widehat{\mathbb{G}})
$$

is given by

$$
m(X)=W_{\mathbb{G}^{\mathrm{op}}} X W_{\mathbb{G}^{\mathrm{op}}}^{*},
$$

where $W_{\mathbb{G}^{\circ}}$ is the left multiplicative unitary of the opposite quantum group $\mathbb{G}^{\text {op }}$ obtained from $\mathbb{G}$. The resulting double crossed product quantum group is precisely Drinfeld's quantum double, as has been shown in [4, Section 8]. Moreover, by [4, Proposition 8.1], Drinfeld's quantum doubles are always unimodular. It also follows from [4, Theorem 5.3] that if $\mathbb{G}$ has non-trivial scaling group, then the double crossed product has non-trivial scaling group as well. Thus Drinfeld's quantum double construction produces concrete examples of unimodular locally compact quantum groups with non-trivial scaling group, and by Theorem 4.3 the Admissibility Conjecture is true for such quantum groups.

\section{Characterisation of Kazhdan's Property T For locally COMPaCt Quantum GROUPS}

Let $A$ be a $\mathrm{C}^{*}$-algebra and let $\pi: A \rightarrow B\left(H_{\pi}\right)$ and $\rho: A \rightarrow B\left(H_{\rho}\right)$ be two non-degenerate representations. The representation $\pi$ is said to be equivalent to $\rho$ if there exists a unitary $U: H_{\pi} \rightarrow H_{\rho}$ such that $U^{*} \rho(x) U=\pi(x)$ for every $x \in A$. If $U$ is only an isometry, then we will say that $\pi$ is contained in $\rho$ and write $\pi \subset \rho$ (in other words, $\pi$ is a subrepresentation of $\rho$ ). Now let $\mathcal{S}$ be a set of representations of $A$. We say that the representation $\pi$ is weakly 
contained in $\mathcal{S}$ and write $\pi \prec \mathcal{S}$ if $\bigcap_{\rho \in \mathcal{S}} \operatorname{Ker} \rho \subset \operatorname{Ker} \pi$. We will adopt the convention that whenever $\pi \prec\{\rho\}$ we will simply write $\pi \prec \rho$.

Let $\widehat{A}$ denote the set of inequivalent irreducible representations of $A$. The closure of $\mathcal{S} \subset \widehat{A}$ is defined as

$$
\overline{\mathcal{S}}=\{\pi \in \widehat{A}: \pi \prec \mathcal{S}\} .
$$

From [21, Lemma 1.6] it follows that the above closure defines a topology on $\widehat{A}$, which is referred to as the Fell topology on $\widehat{A}$ (in [21] this was called the hull-kernel topology on $\widehat{A}$ ). The following result from [10, Lemma 2.1] is crucial in the sequel.

Proposition 5.1. Let $A$ be a $C^{*}$-algebra. If $\rho \in \widehat{A}$ is finite-dimensional, then $\{\rho\}$ is a closed subset of $\widehat{A}$. Moreover, the following statements are equivalent for $\rho \in \widehat{A}$ :

(i) $\rho$ is an isolated point in $\widehat{A}$.

(ii) If a representation $\pi$ of $A$ satisfies $\rho \prec \pi$, then $\rho \subset \pi$.

(iii) $A=\operatorname{Ker} \rho \oplus \bigcap_{\nu \in \widehat{A} \backslash\{\rho\}} \operatorname{Ker} \nu$.

Remark 5.2. It is worthwhile to mention that recently in [9, Definition 3.3] the authors have used a slightly different notion of weak containment, namely a $\mathrm{C}^{*}$-algebraic version of $[50$, Definition 7.3.5]. However, we will be concerned with irreducible representations in which case the definition coincide (as mentioned after Definition 3.3 in 9]).

The following definition is a natural extension of containment to the setting of unitary representations of locally compact quantum groups (see [16, Definition 3.2]).

Definition 5.3. Let $U \in M\left(C_{0}(\mathbb{G}) \otimes \mathcal{K}(H)\right)$ and $V \in M\left(C_{0}(\mathbb{G}) \otimes \mathcal{K}(K)\right)$ be two unitary representations of a locally compact quantum group $\mathbb{G}$, and let $\pi_{U}: C_{0}^{u}(\widehat{\mathbb{G}}) \rightarrow B(H)$ and $\pi_{V}: C_{0}^{u}(\widehat{\mathbb{G}}) \rightarrow B(K)$ be the associated representations of $C_{0}^{u}(\widehat{\mathbb{G}})$ (i.e. $U=\left(\iota \otimes \pi_{U}\right)(\mathrm{W})$ and $\left.V=\left(\iota \otimes \pi_{V}\right)(\mathrm{W})\right)$. If $\pi_{U} \prec \pi_{V}$, we say $U$ is weakly contained in $V$ and write $U \prec V$. If $\pi_{U} \subset \pi_{V}$, we say that $U$ is contained in $V$ (or that $U$ is a subrepresentation of $V$ ) and write $U \subset V$.

Remark 5.4. Let $U \prec V$, so that $\pi_{U} \prec \pi_{V}$. Let $W$ be a corepresentation. We claim that $W \oplus U \prec W \Subset V$. Indeed, this is equivalent to $\pi_{W \bigoplus U}=\left(\pi_{W} \otimes \pi_{U}\right) \circ \chi \circ \widehat{\Delta}_{u} \prec\left(\pi_{W} \otimes \pi_{V}\right) \circ$ $\chi \circ \widehat{\Delta}_{u}=\pi_{W(V}$, which by definition, is equivalent to showing that $\operatorname{ker}\left(\pi_{W} \otimes \pi_{V}\right) \circ \widehat{\Delta}_{u} \subseteq$ $\operatorname{ker}\left(\pi_{W} \otimes \pi_{U}\right) \circ \widehat{\Delta}_{u}$.

Let $W$ act on $H_{W}$, and let $a \in \operatorname{ker}\left(\pi_{W} \otimes \pi_{V}\right) \circ \widehat{\Delta}_{u}$. For $\omega \in \mathcal{K}\left(H_{W}\right)^{*}$ let $b=\left(\omega \circ \pi_{W} \otimes \iota\right) \widehat{\Delta}_{u}(a)$, so that $\pi_{V}(b)=0$. As $\pi_{U} \prec \pi_{V}$, it follows that $\pi_{U}(b)=0$, so that $(\omega \otimes \iota)\left(\pi_{W} \otimes \pi_{U}\right) \circ \widehat{\Delta}_{u}(a)=0$. As $\omega$ was arbitrary, $\left(\pi_{W} \otimes \pi_{U}\right) \circ \widehat{\Delta}_{u}(a)=0$, as required.

We next recall the definitions of invariant and almost invariant vectors for quantum group representations (see [16, Definition 3.2]).

Definition 5.5. Let $U \in M\left(C_{0}(\mathbb{G}) \otimes \mathcal{K}(H)\right)$ be a representation of a locally compact quantum group $\mathbb{G}$. A vector $\xi \in H$ is called invariant for $U$ if $U(\eta \otimes \xi)=\eta \otimes \xi$ for all $\eta \in L^{2}(\mathbb{G})$. $U$ is said to have almost invariant vectors if there exits a net $\left(\xi_{\alpha}\right)_{\alpha}$ of unit vectors in $H$ such that $\left\|U\left(\eta \otimes \xi_{\alpha}\right)-\eta \otimes \xi_{\alpha}\right\| \rightarrow 0$ for all $\eta \in L^{2}(\mathbb{G})$.

Note that if $U$ and $V$ are similar representations of a locally compact quantum group $\mathbb{G}$, then $U$ has an invariant vector if and only if $V$ has. The analogous statement holds for almost invariant vectors. 
We will need invariant means in the following arguments, so next we set up the necessary terminology for those.

Definition 5.6. The (reduced) Fourier-Stieltjes algebra of $\mathbb{G}$ is defined by

$$
B(\mathbb{G})=\operatorname{span}\left\{(\iota \otimes \omega)(\mathrm{W}): \omega \in C_{0}^{u}(\widehat{\mathbb{G}})^{*}\right\} .
$$

Then the Eberlein algebra of $\mathbb{G}$ is defined by

$$
E(\mathbb{G})=\overline{B(\mathbb{G})}\|\cdot\|_{B\left(L^{2}(\mathbb{G})\right)} .
$$

Note that $B(\mathbb{G}) \subset M\left(C_{0}(\mathbb{G})\right)$ and that $B(\mathbb{G})$ is a subalgebra of $M\left(C_{0}(\mathbb{G})\right)$. It then follows that $E(\mathbb{G})$ is a closed subalgebra of $M\left(C_{0}(\mathbb{G})\right)$. When $\mathbb{G}$ is of Kac type, $E(\mathbb{G})$ is self-adjoint and so a $\mathrm{C}^{*}$-subalgebra of $M\left(C_{0}(\mathbb{G})\right.$ ) (see for example [14, Section 7]).

The Banach algebra $L^{1}(\mathbb{G})$ acts on its dual $L^{\infty}(\mathbb{G})$ by

$$
x \cdot \omega=(\omega \otimes \iota)(\Delta(x)), \quad \omega \in L^{1}(\mathbb{G}), x \in L^{\infty}(\mathbb{G}),
$$

and

$$
\omega \cdot x=(\iota \otimes \omega)(\Delta(x)), \quad \omega \in L^{1}(\mathbb{G}), x \in L^{\infty}(\mathbb{G}),
$$

making $L^{\infty}(\mathbb{G})$ an $L^{1}(\mathbb{G})$-bimodule. It is easy to see that $E(\mathbb{G})$ is invariant under these actions. This allows us to define the notion of an invariant mean on $E(\mathbb{G})$. It follows from [18. Proposition 3.15] that $E(\mathbb{G})$ admits an invariant mean $\mu \in E(\mathbb{G})^{*}$ in the sense that $\|\mu\|=\mu(1)=1$ and

$$
\mu(\omega \cdot x)=\omega(1) \mu(x)=\mu(x \cdot \omega) \quad \omega \in L^{1}(\mathbb{G}), x \in E(\mathbb{G}) .
$$

Note that the Hopf $*$-algebra $\mathcal{A P}(\mathbb{G})$ underlying the quantum Bohr compactification is contained in $B(\mathbb{G})$, and therefore $A P(\mathbb{G}) \subset E(\mathbb{G})$. The uniqueness of the Haar state of a compact quantum group implies the following result.

Lemma 5.7. Let $M$ be the restriction of the invariant mean $\mu \in E(\mathbb{G})^{*}$ to $A P(\mathbb{G})$. Then $M$ is the Haar state of the compact quantum group $(A P(\mathbb{G}), \Delta)$.

Lemma 5.8. Let $X \in B\left(L^{2}(\mathbb{G})\right) \bar{\otimes} B(H)$ for some Hilbert space $H$, and suppose that $(\iota \otimes$ $\omega)(X) \in E(\mathbb{G})$ for all $\omega \in B(H)_{*}$. Let $\nu \in E(\mathbb{G})^{*}$. Then there exists an operator $T \in B(H)$ such that for all $\omega \in B(H)_{*}$

$$
\langle T, \omega\rangle=\langle\nu,(\iota \otimes \omega)(X)\rangle .
$$

We will be denoting this operator by $(\nu \otimes \iota)(X)$, so that

$$
\omega((\nu \otimes \iota)(X))=\nu((\iota \otimes \omega)(X))
$$

for all $\omega \in B(H)_{*}$.

Proof. The map

$$
\omega \mapsto\langle\nu,(\iota \otimes \omega)(X)\rangle: B(H)_{*} \rightarrow \mathbb{C}
$$

defines a bounded functional on $B(H)_{*}$, which gives the existence of the operator $T \in B(H)$.

The next result gives a formula for the orthogonal projection onto the set of invariant vectors for a unitary corepresentation of $C_{0}(\mathbb{G})$ (also see [18, Proposition 3.14]).

Lemma 5.9. Let $V \in M\left(C_{0}(\mathbb{G}) \otimes \mathcal{K}(H)\right)$ be a unitary representation of $\mathbb{G}$ and let $\mu \in E(\mathbb{G})^{*}$ be the invariant mean on $E(\mathbb{G})$. The operator $P=(\mu \otimes \iota)(V) \in B(H)$ is a projection onto the subspace of invariant vectors for $V$. 
Proof. Due to the subtle definition of $(\mu \otimes \iota)(V)$, we include a careful calculation of the fact that the image of $P$ consists of invariant vectors. Given $\sigma \in L^{1}(\mathbb{G})$ and $\omega \in B(H)_{*}$, we have

$$
(\sigma \otimes \omega)(V(1 \otimes P))=\omega((\sigma \otimes \iota)(V) P)=\mu\left((\sigma \otimes \iota \otimes \omega)\left(V_{13} V_{23}\right)\right)
$$

due to the commutation relation in Lemma 5.8. Continuing from here using the fact that $V$ is a representation, we have

$$
\begin{aligned}
(\sigma \otimes \omega)(V(1 \otimes P)) & =\mu((\sigma \otimes \iota \otimes \omega)((\Delta \otimes \iota)(V)))=\mu((\sigma \otimes \iota)(\Delta((\iota \otimes \omega)(V)))) \\
& =\sigma(1) \mu((\iota \otimes \omega)(V))=(\sigma \otimes \omega)(1 \otimes P)
\end{aligned}
$$

due to the invariance of $\mu$. It follows that the image of $P$ consists of invariant vectors. That $P$ is an idempotent map is also easy to compute. Notice that $\|P\| \leq 1$, because $\|\mu\|=1$, from which it follows that $P$ is the orthogonal projection onto the subspace of invariant vectors.

The following result from [16. Corollary 2.5 and Corollary 2.8] gives a nice criterion for the existence of invariant and almost invariant vectors. Denote the trivial representation of $\mathbb{G}$ by 1 .

Proposition 5.10. Let $U \in M\left(C_{0}(\mathbb{G}) \otimes \mathcal{K}(H)\right)$ be a unitary representation of a locally quantum group $\mathbb{G}$.

(i) $U$ has a nonzero invariant vector if and only if $1 \subset U$.

(ii) $U$ has almost invariant vectors if and only if $1 \prec U$.

We now recall the definition of Kazhdan's Property (T) for quantum groups (see [10, Definition 3.1], which goes back to [22, Definition 6]).

Definition 5.11. A locally compact quantum group $\mathbb{G}$ has (Kazhdan's) Property $(T)$ if every unitary representation of $\mathbb{G}$ that has almost invariant vectors has a nonzero invariant vector.

It follows from Proposition 5.10 that $\mathbb{G}$ has Property $(\mathrm{T})$ if and only if for every unitary representation $U$ of $\mathbb{G}$

$$
1 \prec U \Longrightarrow 1 \subset U \text {. }
$$

The following theorem [10, Proposition 3.2 and Theorem 3.6] and [9, Theorem 4.7] gives a series of equivalent conditions to Property (T).

Theorem 5.12. Let $\mathbb{G}$ be a locally compact quantum group. The following statements are equivalent:

(T1) $\mathbb{G}$ has Property (T).

(T2) The counit $\widehat{\epsilon}_{u}$ is an isolated point in $\widehat{C_{0}^{u(\widehat{\mathbb{G}})}}$.

(T3) $C_{0}^{u}(\widehat{\mathbb{G}})=\operatorname{Ker} \widehat{\epsilon}_{u} \oplus \mathbb{C}$.

(T4) There is a projection $p \in M\left(C_{0}^{u}(\widehat{\mathbb{G}})\right)$ such that $p C_{0}^{u}(\widehat{\mathbb{G}}) p=\mathbb{C} p$ and $\widehat{\epsilon}_{u}(p)=1$.

If $\mathbb{G}$ has trivial scaling group, then the above conditions are further equivalent to the following (quantum version of Wang's theorem [44, Theorem 2.1]):

(T5) Every finite-dimensional irreducible representation of $C_{0}^{u}(\widehat{\mathbb{G}})$ is an isolated point in $\widehat{C_{0}^{u}(\widehat{\mathbb{G}})}$.

(T6) $C_{0}^{u}(\widehat{\mathbb{G}}) \cong B \oplus M_{n}(\mathbb{C})$ for some $C^{*}$-algebra $B$ and $n \in \mathbb{N}_{0}$. 
We will prove that for a locally compact quantum group with non-trivial scaling group, (T5) as well as a suitable generalisation of (T6) are equivalent with (T1)-(T4).

We first make two observations, which will be used later (also see [43, Proposition 3.14 \& Proposition 3.15]).

Lemma 5.13. Let $U \in M\left(C_{0}(\mathbb{G}) \otimes M_{n}(\mathbb{C})\right)$ be a finite-dimensional admissible unitary representation of $\mathbb{G}$. Then $1 \subset U^{c} \uparrow U$.

Proof. Write $U=\left(U_{i j}\right)_{i, j=1}^{n}$, and define $\bar{U}=\left(U_{i j}^{*}\right)_{i, j=1}^{n}$. Since $U$ is admissible, $\bar{U} \in E(\mathbb{G}) \otimes$ $M_{n}(\mathbb{C})$. Define $V=\bar{U} \oplus U \in E(\mathbb{G}) \otimes\left(M_{n}(\mathbb{C}) \otimes M_{n}(\mathbb{C})\right)$. Let $\mu$ be the invariant mean on $E(\mathbb{G})$. We will show that $(\mu \otimes \iota)(V) \neq 0$, where we are using the notation of Lemma 5.8. Towards a contradiction, suppose that $(\mu \otimes \iota)(V)=0$, so that for all $\nu \in\left(M_{n}(\mathbb{C}) \otimes M_{n}(\mathbb{C})\right)_{*}$ we have $\mu((\iota \otimes \nu)(V))=0$. Choosing $\nu$ such that $(\iota \otimes \nu)(V)=U_{i j}^{*} U_{i j}$ leads to $\mu\left(U_{i j}^{*} U_{i j}\right)=0$ for all $i, j=1,2, \ldots, n$. Therefore,

$$
\mu\left(\sum_{i=1}^{n} U_{i j}^{*} U_{i j}\right)=\mu(1)=0
$$

which cannot happen as $\mu(1)=1$.

By Lemma [5.9, any vector in the image of $(\mu \otimes \iota)(V)$ is an invariant vector for $V$. Since $\bar{U}$ is similar to $U^{c}$ (as $U$ is admissible, Remark (3.5), the representation $V$ is similar to $U^{c}(T)$ and the result follows from Proposition 5.10 - (i).

The following result may be considered as an extension of [10, Proposition 3.5] (see also [8, Proposition A.1.12] and [29, Theorem 2.6]).

Theorem 5.14. Let $U \in M\left(C_{0}(\mathbb{G}) \otimes M_{n}(\mathbb{C})\right)$ and $V \in M\left(C_{0}(\mathbb{G}) \otimes \mathcal{K}(H)\right)$ be unitary representations of a locally compact quantum group $\mathbb{G}$ with $U$ being admissible. Then the following are equivalent:

(i) The representations $U$ and $V$ contain a common finite-dimensional unitary representation.

(ii) The representation $U^{c} \uparrow V$ contains the trivial representation.

Proof. (i) $\Longrightarrow$ (ii): Let $W$ be a common finite-dimensional unitary representation of $U$ and $V$. Then $W$ is also admissible as $U$ is admissible. We have $W^{c} \oplus W \subset U^{c} \uparrow V$ and by Lemma 5.13, $1 \subset W^{c} \uparrow W$.

(ii) $\Longrightarrow$ (i): Let $\mu$ denote the invariant mean on $E(\mathbb{G})$. Let $x \in B\left(H, \mathbb{C}^{n}\right)$ and $y=$ $(\mu \otimes \iota)\left(U^{*}(1 \otimes x) V\right)$ so that $y \in B\left(H, \mathbb{C}^{n}\right)$. Now

$$
\begin{aligned}
U^{*}(1 \otimes y) V & =(\mu \otimes \iota \otimes \iota)\left(U_{23}^{*} U_{13}^{*}(1 \otimes 1 \otimes x) V_{13} V_{23}\right) \\
& =(\mu \otimes \iota \otimes \iota) \circ(\Delta \otimes \iota)\left(U^{*}(1 \otimes x) V\right)=1 \otimes y
\end{aligned}
$$

due to the invariance of $\mu$ (see the proof of Lemma 5.9 for making the above calculation more rigorous). Since $U$ is unitary, we have

$$
U(1 \otimes y)=(1 \otimes y) V .
$$

Next we show that for some $x$, the resulting $y$ is nonzero,

It follows from the hypothesis, via Proposition 5.10 (i), that $\bar{U} \odot V$ has an invariant vector $\zeta \in \mathbb{C}^{n} \otimes H$, and so

$$
\left(\iota \otimes \omega_{\zeta, \zeta}\right)(\bar{U} \odot V)=\langle\zeta, \zeta\rangle 1
$$


For $\xi \in H$ and $\alpha \in \mathbb{C}^{n}$, let $x=\theta_{\alpha, \xi} \in B\left(H, \mathbb{C}^{n}\right)$ be defined by $\theta_{\alpha, \xi}(\eta)=\langle\eta, \xi\rangle \alpha$ for $\eta \in H$. If $y=(\mu \otimes \iota)\left(U^{*}\left(1 \otimes \theta_{\alpha, \xi}\right) V\right)=0$ for every $\xi \in H$ and $\alpha \in \mathbb{C}^{n}$, then for every $\alpha, \beta \in \mathbb{C}^{n}$ and $\xi, \eta \in H$, we have

$$
\begin{aligned}
0=\langle y \eta, \beta\rangle & =\mu\left(\left(\iota \otimes \omega_{\eta, \beta}\right)\left(U^{*}\left(1 \otimes \theta_{\alpha, \xi}\right) V\right)\right)=\mu\left(\left(\iota \otimes \omega_{\beta, \alpha}\right)(U)^{*}\left(\iota \otimes \omega_{\eta, \xi}\right)(V)\right) \\
& =\mu\left(\left(\iota \otimes \omega_{\alpha, \beta}\right)(\bar{U})\left(\iota \otimes \omega_{\eta, \xi}\right)(V)\right) .
\end{aligned}
$$

Therefore $(\mu \otimes \iota)(\bar{U} \odot V)=0$, and so by equation (5)

$$
0=\omega_{\zeta, \zeta}((\mu \otimes \iota)(\bar{U} \odot V))=\mu\left(\left(\iota \otimes \omega_{\zeta, \zeta}\right)(\bar{U} \odot V)\right)=\langle\zeta, \zeta\rangle \mu(1) \neq 0 .
$$

Consequently, $y \neq 0$ for some $\xi \in H, \alpha \in \mathbb{C}^{n}$.

To finish the proof we may argue as in the last part of the proof of [29, Theorem 2.6].

We now prove a generalisation of conditions (T5) and (T6) in Theorem 5.12.

Theorem 5.15. Let $\mathbb{G}$ be any locally compact quantum group. Then $\mathbb{G}$ having Property $(T)$ is equivalent to any of the following statements:

(T5) Every finite-dimensional irreducible $C^{*}$-representation of $C_{0}^{u}(\widehat{\mathbb{G}})$ is an isolated point in $\widehat{C_{0}^{u}(\widehat{\mathbb{G}})}$.

$\left(\mathrm{T} 6^{\prime}\right)$ There is a finite-dimensional irreducible $C^{*}$-representation of $C_{0}^{u}(\widehat{\mathbb{G}})$ which is covariant with respect to the scaling automorphism group $\left(\widehat{\tau}_{t}^{u}\right)$ and is an isolated point in $\widehat{C_{0}^{u}(\widehat{\mathbb{G}})}$.

(T6) $C_{0}^{u}(\widehat{\mathbb{G}}) \cong B \oplus M_{n}(\mathbb{C})$ for some $C^{*}$-algebra $B$ and some $n \in \mathbb{N}_{0}$ with $\widehat{\tau}_{t}^{u}(B) \subset B$ for all $t \in \mathbb{R}$.

Proof. The proof is based on the same idea as the proof of [10, Theorem 3.6].

$(\mathrm{T} 5) \Longrightarrow\left(\mathrm{T} 6^{\prime}\right)$ because covariant irreducible finite-dimensional representations always exist: the counit.

$\left(\mathrm{T} 6^{\prime}\right) \Longrightarrow(\mathrm{T} 6)$ : Let $\pi$ be a finite-dimensional irreducible representation of $C_{0}^{u}(\widehat{\mathbb{G}})$ which is covariant with respect to the scaling automorphism group. By the equivalence of (i) and (iii) in Proposition 5.1. $C_{0}^{u}(\widehat{\mathbb{G}}) \cong \operatorname{ker} \pi \oplus M_{n}(\mathbb{C})$ for some $n \in \mathbb{N}_{0}$. Since $\pi$ is covariant, we have $\widehat{\tau}_{t}^{u}(\operatorname{ker}(\pi)) \subset \operatorname{ker} \pi$ for all $t \in \mathbb{R}$, and so (T6) holds.

It remains to prove that (T1) of Theorem 5.12 implies (T5) and that (T6) implies (T2) of Theorem 5.12. The result will then follow from the equivalence of (T1) and (T2) of Theorem 5.12 ,

$(\mathrm{T} 1) \Longrightarrow(\mathrm{T} 5)$ : To prove (T5), it is enough to show that (ii) in Proposition 5.1 holds for every irreducible finite-dimensional representation $\rho$ of $C_{0}^{u}(\widehat{\mathbb{G}})$. To this end, let $\pi$ be a representation of $C_{0}^{u}(\widehat{\mathbb{G}})$ such that $\rho \prec \pi$. Let $U=(\iota \otimes \rho)(\mathrm{W})$ and $V=(\iota \otimes \pi)(\mathrm{W})$. Since $\mathbb{G}$ has Property $(\mathrm{T})$, it is unimodular by Theorem 6.1 and so $\mathbb{G}$ satisfies the Admissibility Conjecture by Theorem 4.3. Hence $U$ is an irreducible finite-dimensional admissible unitary representation of $\mathbb{G}$ and $U \prec V$. Now by Lemma 5.13 we have that $1 \subset U^{c} \uparrow U$ and also $U^{c} \uparrow U \prec U^{c} \uparrow V$ by Remark 5.4. Since $\mathbb{G}$ has Property $(\mathrm{T})$ and $1 \prec U^{c} \uparrow V$, it follows that $1 \subset U^{c} \uparrow V$. Thus by Theorem 5.14 there exists a finite-dimensional unitary representation $W$ such that $W \subset U$ and $W \subset V$. Since $U$ is irreducible, this implies that $W=U$, and so $\rho \subset \pi$.

$(\mathrm{T} 6) \Longrightarrow(\mathrm{T} 2)$ : Suppose that $C_{0}^{u}(\widehat{\mathbb{G}}) \cong B \oplus M_{n}(\mathbb{C})$ and $B$ in invariant under the scaling automorphism group. Let $\mu: C_{0}^{u}(\widehat{\mathbb{G}}) \rightarrow M_{n}(\mathbb{C})$ be the irreducible representation corresponding to the summand $M_{n}(\mathbb{C})$, and let $U=(\iota \otimes \mu)(\mathrm{W})$ be the unitary representation of $\mathbb{G}$ 
associated with $\mu$. Since $\operatorname{ker}(\mu) \cong B$ and $\widehat{\tau}_{t}^{u}(B) \subset B$ for all $t \in \mathbb{R}$, it follows that $\mu$ is covariant with respect to the scaling automorphism group of $C_{0}^{u}(\widehat{\mathbb{G}})$. Hence $U$ is admissible by Proposition 3.2. The representation $\pi$ of $C_{0}^{u}(\widehat{\mathbb{G}})$ associated to $U^{c} \uparrow U$ is finite-dimensional and covariant with respect to scaling automorphism of $C_{0}^{u}(\widehat{\mathbb{G}})$ by Proposition 3.2 since $U^{c} \uparrow U$ is admissible. Therefore, $\pi$ decomposes into a direct sum of finitely many covariant irreducible representations. Since $1 \subset U^{c} \uparrow U$ by Lemma 5.13, one of the irreducible components is $\widehat{\epsilon}_{u}$. Therefore $\pi=\bigoplus_{k=0}^{m} \pi_{k}$, where $\pi_{0}=\widehat{\epsilon}_{u}$ and $\pi_{k}$ is an irreducible finite-dimensional covariant representation for each $k=1,2, \ldots, m$. By Proposition [5.1, each singleton set $\left\{\pi_{k}\right\}$ is closed in the Fell topology. Towards a contradiction, let us assume that $\widehat{\epsilon}_{u}$ is not an isolated point. So there is a net

$$
\left(\rho_{\alpha}\right)_{\alpha \in \Lambda} \subset \widehat{C_{0}^{u}(\widehat{\mathbb{G}})} \backslash\left\{\widehat{\epsilon}_{u}, \pi_{1}, \pi_{2}, \ldots, \pi_{m}\right\}
$$

such that $\left(\rho_{\alpha}\right)_{\alpha \in \Lambda}$ converges in the Fell topology to $\widehat{\epsilon}_{u}$. By the definition of closure in the Fell topology, this implies that $\widehat{\epsilon}_{u} \prec \bigoplus_{\alpha \in \Lambda} \rho_{\alpha}$, and so

$$
\mu=\left(\mu \otimes \widehat{\epsilon}_{u}\right) \circ \chi \circ \widehat{\Delta}_{u} \prec \bigoplus_{\alpha \in \Lambda}\left(\mu \otimes \rho_{\alpha}\right) \circ \chi \circ \widehat{\Delta}_{u},
$$

where $\chi$ is the flip map. Since $\mu$ satisfies condition (iii) in Proposition 5.1 (by definition), it follows by condition (ii) in Proposition 5.1 that

$$
\mu \subset \bigoplus_{\alpha \in \Lambda}\left(\mu \otimes \rho_{\alpha}\right) \circ \chi \circ \widehat{\Delta}_{u} .
$$

By irreducibility of $\mu$ we have $\mu \subset\left(\mu \otimes \rho_{\alpha}\right) \circ \chi \circ \widehat{\Delta}_{u}$ for some $\alpha \in \Lambda$. Letting $U_{\alpha}=\left(\iota \otimes \rho_{\alpha}\right)($ W), this means that $U \subset U \odot U_{\alpha}$. Combining this with Lemma 5.13 it follows that

$$
1 \subset U^{c} \oplus U \subset U^{c} \uparrow U \oplus U_{\alpha},
$$

so that we have

$$
1 \subset U^{c} \oplus U \oplus U_{\alpha}=\left(\bigoplus_{k=0}^{m}\left(\iota \otimes \pi_{k}\right)(\mathrm{W})\right) \oplus U_{\alpha} .
$$

Since $\left(U^{c} \odot U\right)^{c}$ is equivalent to $U^{c} \uparrow U$ (recall that $U$ is admissible, Remark [3.5), it follows that

$$
1 \subset\left(\bigoplus_{k=0}^{m}\left(\iota \otimes \pi_{k}\right)(\mathrm{W})\right)^{c} \oplus U_{\alpha} .
$$

An application of Theorem 5.14 now yields a finite-dimensional unitary representation $W$ such that $W \subset \bigoplus_{k=0}^{m}\left(\iota \otimes \pi_{k}\right)(\mathrm{W})$ and $W \subset U_{\alpha}$. Since $U_{\alpha}$ is irreducible, we have $W=U_{\alpha}$. On the other hand, $\pi_{k}$ is irreducible for $k=0,1,2, \ldots, m$, and so $U_{\alpha}=\left(\iota \otimes \pi_{k_{0}}\right)(\mathrm{W})$ for some $k_{0} \in\{0,1,2, \ldots, m\}$. This means that $\rho_{\alpha}=\pi_{k_{0}}$ which is a contradiction. Thus $\widehat{\epsilon}_{u}$ must be an isolated point.

\section{Properties of quantum groups with Property (T)}

In this section we prove several properties shared by quantum groups with Property $(\mathrm{T})$. We include a very short proof of the fact that a quantum group with Property (T) is unimodular (see [9, Section 6] and [22, Proposition 7]). We consider unimodular locally compact quantum groups as well as quantum groups arising through the bicrossed product construction as discussed in Subsection 4.1 and prove a variation of Theorem 5.15 and improved versions of the quantum versions of Bekka-Valette theorem [9, Theorem 4.8] (characterising Property 
(T) in terms of non-existence of almost invariant vectors for weakly mixing representations) and Kerr-Pichot theorem [9, Theorem 4.9] (characterising Property (T) in terms of density properties of weakly mixing representations) for these quantum groups.

6.1. Quantum groups with Property $(\mathbf{T})$ are unimodular. It is a well-known fact that a locally compact group $G$ with Property $(\mathrm{T})$ is unimodular [8, Corollary 1.3.6-(ii)]. The proof of this result makes use of the fact that if $G$ has Property (T) and admits a continuous homomorphism into a locally compact group $H$ with dense range, then $H$ has Property (T) 8 , Theorem 1.3.4]. A version of [8, Theorem 1.3.4] for locally compact quantum groups has been obtained in [18, Theorem 5.7]. Using this, it seems plausible that one can prove that Property (T) for quantum groups implies unimodularity similarly to the classical case. However, the proofs in the case of quantum groups have proceeded differently.

To the best of our knowledge, the first result in this direction for quantum groups was proven for discrete quantum groups [22, Proposition 7]. This was subsequently generalised to second countable locally compact quantum groups [9, Section 6]. The proof in the case of a locally compact quantum group, as given in [9], proceeds via showing that non-unimodular locally compact quantum groups always admit a weakly mixing representation that weakly contains the trivial representation, as a consequence the quantum group cannot have Property ( $\mathrm{T})$.

We give a very short proof of the fact that Property (T) implies unimodularity, using a completely different technique, which does not require the second countability assumption.

Theorem 6.1. Let $\mathbb{G}$ be a locally compact quantum group with Property (T). Then $\mathbb{G}$ is unimodular.

Proof. Let $\delta$ denote the modular element of $\mathbb{G}$, so that $\delta$ is a strictly positive element affiliated to $C_{0}(\mathbb{G})$ [27, Definition 7.11]. By [27, Proposition 7.12], for all $s, t \in \mathbb{R}$,

(i) $\Delta\left(\delta^{i s}\right)=\delta^{i s} \otimes \delta^{i s}$,

(ii) $\tau_{t}\left(\delta^{i s}\right)=\delta^{i s}$.

Note that $\delta^{0}=1$ is the trivial representation of $\mathbb{G}$. Relation (i) implies that for all $s \in \mathbb{R}, \delta^{i s}$ is a 1-dimensional unitary representation of $\mathbb{G}$, so there exists $\mathrm{C}^{*}$-representations $\pi_{s}: C_{0}^{u}(\widehat{\mathbb{G}}) \rightarrow$ $\mathbb{C}$ such that $\left(\iota \otimes \pi_{s}\right)(\mathrm{W})=\delta^{i s}$. For all $\omega \in B\left(L^{2}(\mathbb{G})\right)_{*}$, it follows that

$$
\lim _{s \rightarrow 0} \pi_{s}((\omega \otimes \iota)(\mathrm{W}))=\lim _{s \rightarrow 0} \omega\left(\delta^{i s}\right)=\omega(1)=\widehat{\epsilon}_{u}((\omega \otimes \iota)(\mathrm{W})),
$$

where $\widehat{\epsilon}_{u}$ is the counit of $C_{0}^{u}(\widehat{\mathbb{G}})$. Since the family $\left\{\pi_{s}\right\}_{s \in \mathbb{R}}$ is uniformly bounded and the elements of the form $(\omega \otimes \iota)(\mathrm{W})$ for $\omega \in B\left(L^{2}(\mathbb{G})\right)_{*}$ are norm-dense in $C_{0}^{u}(\widehat{\mathbb{G}})$ [26, Equation (5.2)], it follows that $\lim _{s \rightarrow 0} \pi_{s}(x)=\widehat{\epsilon}_{u}(x)$ for all $x \in C_{0}^{u}(\widehat{\mathbb{G}})$.

By [26, Proposition 9.1], $\left(\tau_{t} \otimes \iota\right)(\mathrm{W})=\left(\iota \otimes \widehat{\tau}_{-t}^{u}\right) \mathrm{W}$ for all $t \in \mathbb{R}$, where $\left(\widehat{\tau}_{t}^{u}\right)_{t \in \mathbb{R}}$ is the universal scaling group of $\widehat{\mathbb{G}}$. It then follows by relation (ii) above that $\pi_{t}\left(\widehat{\tau}_{s}^{u}(x)\right)=\pi_{t}(x)$ for all $s, t \in \mathbb{R}$ and $x \in C_{0}^{u}(\widehat{\mathbb{G}})$, so that for each $t \in \mathbb{R}, \pi_{t}: C_{0}^{u}(\widehat{\mathbb{G}}) \rightarrow \mathbb{C}$ is covariant in the sense of Remark 3.4. Since $\mathbb{G}$ has Property $(\mathrm{T})$ by hypothesis, $\widehat{\epsilon}_{u}$ is isolated in $\widehat{C_{0}^{u}(\widehat{\mathbb{G}})}$. Since $\lim _{t \rightarrow 0} \pi_{t}(x)=\widehat{\epsilon}_{u}(x)$ for all $x \in C_{0}^{u}(\widehat{\mathbb{G}})$, it follows that the net $\left(\pi_{t}\right)_{t \in \mathbb{R}}$ converges to $\widehat{\epsilon}_{u}$ in the Fell topology of $\widehat{C_{0}^{u}(\widehat{\mathbb{G}})}$. As $\widehat{\epsilon}_{u}$ is isolated, we must have $\pi_{t}=\widehat{\epsilon}_{u}$ for all $t \in \mathbb{R}$ with $|t|$ sufficiently small, that is, $\delta^{i t}=1$ for all $t \in \mathbb{R}$ with $|t|$ sufficiently small. It follows that in fact $\delta^{i t}=1$ for all $t \in \mathbb{R}$, and so that $\delta=1$, as required. 
6.2. Other aspects of quantum groups with Property (T). Combining Proposition 3.2, Theorem 4.3 and Theorem 5.15 we have the following.

Corollary 6.2. Let $\mathbb{G}$ be a locally compact quantum group that is either unimodular or arises through the bicrossed product construction as described in Theorem 4.2. Then $\mathbb{G}$ having Property $(T)$ is equivalent to either of the following statements:

$\left(\mathrm{T} 6^{\prime}\right)$ There is a finite-dimensional irreducible $C^{*}$-representation of $C_{0}^{u}(\widehat{\mathbb{G}})$ that is an isolated point in $\widehat{C_{0}^{u}(\widehat{\mathbb{G}})}$.

(T6) $C_{0}^{u}(\widehat{\mathbb{G}}) \cong B \oplus M_{n}(\mathbb{C})$ for some $C^{*}$-algebra $B$ and some $n \in \mathbb{N}_{0}$.

A unitary representation of a locally compact quantum group is weakly mixing if it admits no nonzero admissible finite-dimensional subrepresentation (see [43]). Corollary 6.2 together with [9, Lemma 3.6] gives the Bekka-Valette theorem for all unimodular locally compact quantum groups. This was known before only for quantum groups with trivial scaling automorphism group [9, Theorem 4.8].

Corollary 6.3. Let $\mathbb{G}$ be a second countable locally compact quantum group that is either unimodular or arises through the bicrossed product construction as described in Theorem 4.2. Then $\mathbb{G}$ has Property $(T)$ if and only if every weakly mixing unitary representation of $\mathbb{G}$ fails to have almost invariant vectors.

Combining Corollary 6.3 with [9, Theorems $3.7 \& 3.8$ ] we have the Kerr-Pichot theorem for unimodular quantum groups with non-trivial scaling group. Also this was known before only for the case of quantum groups with trivial scaling group [9, Theorem 4.8].

Given a second countable locally compact quantum group $\mathbb{G}$ and a Hilbert space $H$, denote the set of all unitary representations of $\mathbb{G}$ on $H$ by $\operatorname{Rep}(\mathbb{G}, H)$ and the set of all weakly mixing unitary representations of $\mathbb{G}$ on $H$ by $W(\mathbb{G}, H)$.

Corollary 6.4. Let $\mathbb{G}$ be a second countable locally compact quantum group that is either unimodular or arises through the bicrossed product construction as described in Theorem 4.2. Let $H$ be a separable infinite-dimensional Hilbert space. If $\mathbb{G}$ does not have Property $(T)$, then $W(\mathbb{G}, H)$ is a dense $G_{\delta}$-set in $\operatorname{Rep}(\mathbb{G}, H)$. If $\mathbb{G}$ has Property $(T)$, then $W(\mathbb{G}, H)$ is closed and nowhere dense in $\operatorname{Rep}(\mathbb{G}, H)$.

\section{REFERENCES}

[1] Y. Arano, Unitary spherical representations of Drinfeld doubles, to appear in J. Reine. Angew. Math.

[2] Y. Arano, Comparison of unitary duals of Drinfeld doubles and complex semisimple Lie groups, Comm. Math. Phys. 351 (2017), 1137-1147.

[3] S. Baaj and G. Skandalis, Unitaires multiplicatifs et dualité pour les produits croisés de C*-algèbres, Ann. Sci. École Norm. Sup. 26 (1993), 425-488.

[4] S. Baaj and S. Vaes, Double crossed products of locally compact quantum groups, J. Inst. Math. Jussieu 4 (2005), 135-173.

[5] E. Bédos, R. Conti and L. Tuset, On amenability and co-amenability of algebraic quantum groups and their corepresentations, Canad. J. Math. 57 (2005), 17-60.

[6] E. Bédos and L. Tuset, Amenability and coamenability for locally compact quantum groups, Int. J. Math 14 no. 8 (2003), 865-884.

[7] B. Bekka and A. Valette, Kazhdan's property (T) and amenable representations, Math. Z. 212 (1993), 293-299.

[8] B. Bekka, P. de la Harpe and A. Valette, Kazhdan's Property (T), Cambridge University Press, Cambridge, 2008. 
[9] M. Brannan and D. Kerr, Quantum groups, Property (T), and weak mixing, Comm. Math. Phys., to appear, see https://doi.org/10.1007/s00220-017-3037-0

[10] X. Chen and C. K. Ng, Property T for general locally compact quantum groups, Internat. J. Math. 26, 1550024 (2015).

[11] A. Connes and B. Weiss, Property T and asymptotically invariant sequences, Israel J. Math. 37 (1980), 209-210.

[12] C. D'Antoni and L. Zsidó, Groups of linear isometries on multiplier C*-algebras, Pacific J. Math. 193 (2000), 279-307.

[13] A. V. Daele, Discrete quantum groups, J. Algebra 180, (1996) 431-444.

[14] B. Das and M. Daws, Quantum Eberlein compactifications and invariant means, Indiana Univ. Math. J. 65 (2016), 307-352.

[15] M. Daws, Remarks on the quantum Bohr compactification, Illinois J. Math. 57 (2013), 1131-1171.

[16] M. Daws, P. Fima, A. Skalski and S. White, The Haagerup property for locally compact quantum groups, J. Reine. Angew. Math. 711 (2016), 189-229.

[17] M. Daws and P. Salmi, Completely positive definite functions and Bochner's theorem for locally compact quantum groups, J. Funct. Anal. 264 (2013), 1525-1546.

[18] M. Daws, A. Skalski and A.Viselter, Around property (T) for quantum groups, Comm. Math. Phys. 353 (2017), no. 1, 69-118.

[19] M. Enock and J. M. Schwartz, Kac algebras and duality of locally compact groups, Springer-Verlag, Berlin, 1992.

[20] P. Eymard, L'algèbre de Fourier d'un groupe localement compact, Bull. Soc. Math. France 92 (1964), 181-236.

[21] J. M. G. Fell, The dual spaces of C*-algebras, Trans. Amer. Math. Soc. 94 (1960), 365-403.

[22] P. Fima, Kazhdan's Property T for Discrete Quantum Groups, Internat. J. Math. 21 (2010), 47-65.

[23] E. Glasner and B. Weiss, Kazhdan's property (T) and the geometry of the collection of invarinat measures, Geom. Funct. Anal. 7 (1997), 917-935.

[24] D. Kerr and M. Pichot, Asymptotic abelianness, weak mixing, and property T, J. Reine. Angew. Math. 623 (2008), 213-235.

[25] J. Kustermans, Locally compact quantum groups, Quantum Independent Increment Processes I, Eds. M. Schürmann and U. Franz, Springer-Verlag, Berlin, 2005, pp. 99-180.

[26] J. Kustermans, Locally compact quantum groups in the universal setting, Internat. J. Math. 12 (2001), 289-338.

[27] J. Kustermans and S. Vaes, Locally compact quantum groups, Ann. Sci. École Norm. Sup. 33 (2000), $837-934$.

[28] J. Kustermans and S. Vaes, Locally compact quantum groups in the von Neumann algebraic setting, Math. Scand. 92 (2003), 68-92.

[29] D. Kyed and P. M. Soltan, Property (T) and exotic quantum group norms, J. Noncommut. Geom. 6 (2012), 773-800.

[30] A. Maes and A. Van Daele, Notes on compact quantum groups, Nieuw Arch. Wisk. 16 (1998), 73-112.

[31] T. Masuda, Y. Nakagami and S. L. Woronowicz, A C*-algebraic framework for quantum groups, Internat. J. Math. 14 (2003), 903-1001.

[32] R. Meyer, S. Roy, S. L. Woronowicz, Homomorphisms of quantum groups, Munster J. Math. 5 (2012), $1-24$.

[33] S. Neshveyev and L. Tuset, Compact quantum groups and their representation categories, Société Mathématique de France, Paris, 2013.

[34] S. Neshveyev and M. Yamashita, Drinfeld center and representation theory for moniodal categories, Comm. Math. Phys. 345 (2016), 385-434.

[35] S. Petrescu and M. Joiţa, Property (T) for Kac algebras, Rev. Roumaine Math. Pures. Appl. 37 (1992), $163-178$

[36] G. Pisier, Similarity problems and completely bounded maps. Second, expanded edition. Includes the solution to "The Halmos problem", Springer-Verlag, Berlin, 2001.

[37] S. Popa and S. Vaes, Representation theory for subfactors, $\lambda$-lattices and C*-tensor categories, Comm. Math. Phys. 340 (2015), 1239-1280.

[38] W. Rudin, Functional Analysis, Second edition, McGraw-Hill, Inc., New York, 1991.

[39] P. Soltan, Quantum Bohr compactification, Illinois J. Math. 49 (2005), 1245-1270. 
[40] P. Soltan and S.L. Woronowicz, From multiplicative unitaries to quantum groups II, J. Funct. Anal. 252 (2007), 42-67.

[41] T. Timmermann, An Invitation to Quantum Groups and Duality, EMS Textbooks in Mathematics, (2008).

[42] S. Vaes and L. Vainerman, Extensions of locally compact quantum groups and the bicrossed product construction, Adv. Math. 175 (2003), 1-101.

[43] A. Viselter, Weak mixing for locally compact quantum groups. Ergodic Theory Dynam. Systems 37 (2017), $657-1680$

[44] P. S. Wang, On isolated points in the dual spaces of locally compact groups, Math. Ann. 218 (1975), 19-34.

[45] S. Wang, Free products of compact quantum groups, Comm. Math. Phys. 167 (1995), 671-692.

[46] S. L. Woronowicz, From multiplicative unitaries to quantum groups, Internat. J. Math. 7 (1996), 127-149.

[47] S. L. Woronowicz, Compact quantum groups, Symétries quantiques (Les Houches, 1995), 845-884 NorthHolland, Amsterdam, 1998.

[48] S. L. Woronowicz, Twisted SU(2) group, An example of a noncommutative differential calculus, Publ. Res. Inst. Math. Sci. 23 (1987), 117-181.

[49] S. L. Woronowicz, A remark on compact matrix quantum groups, Lett. Math. Phys. 21 (1991), 35-39.

[50] R. J. Zimmer, Ergodic Theory and Semisimple Groups, Monographs in Mathematics, 81 (1984), Birkhäuser Verlag, Basel.

Department of Mathematical Sciences, University of Oulu, Finland.

Instytut Matematyczny, Uniwersytet Wroceawski, Poland.

E-mail address: biswarup.das@math.uni.wroc.pl

Jeremiah Horrocks Institute, University of Central Lancashire, UK.

E-mail address: matt.daws@cantab.net

Deprtment of Mathematical Sciences, University of Oulu, Finland.

E-mail address: pekka.salmi@oulu.fi 